\title{
Molecular characterization and expression analysis of pitaya (Hylocereus polyrhizus) $H p L R R$ genes in response to Neoscytalidium dimidiatum infection
}

Min Xu, Cheng-Li Liu, Yu Fu, Zhi-Wen Liao, Pan-Yang Guo, Rui Xiong, Yu Cheng, Shuang-Shuang Wei, Jia-Quan Huang ${ }^{*}$ and Hua Tang*

\begin{abstract}
Background: Canker disease caused by Neoscytalidium dimidiatum is a devastating disease resulting in a major loss to the pitaya industry. However, resistance proteins in plants play crucial roles to against pathogen infection. Among resistance proteins, the leucine-rich repeat (LRR) protein is a major family that plays crucial roles in plant growth, development, and biotic and abiotic stress responses, especially in disease defense.

Results: In the present study, a transcriptomics analysis identified a total of 272 LRR genes, 233 of which had coding sequences (CDSs), in the plant pitaya (Hylocereus polyrhizus) in response to fungal Neoscytalidium dimidiatum infection. These genes were divided into various subgroups based on specific domains and phylogenetic analysis. Molecular characterization, functional annotation of proteins, and an expression analysis of the LRR genes were conducted. Additionally, four LRR genes (CL445.Contig4_All, Unigene28_All, CL28.Contig2_All, and Unigene2712_All, which were selected because they had the four longest CDSs were further assessed using quantitative reverse transcription PCR (qRT-PCR) at different fungal infection stages in different pitaya species (Hylocereus polyrhizus and Hylocereus undatus), in different pitaya tissues, and after treatment with salicylic acid (SA), methyl jasmonate (MeJA), and abscisic acid (ABA) hormones. The associated protein functions and roles in signaling pathways were identified. Conclusions: This study provides a comprehensive overview of the HpLRR family genes at transcriptional level in pitaya in response to $\mathrm{N}$. dimidiatum infection, it will be helpful to understand the molecular mechanism of pitaya canker disease, and lay a strong foundation for further research.
\end{abstract}

Keywords: Pitaya, Canker disease, Neoscytalidium dimidiatum, Transcriptomics, Leucine-rich-repeat genes, Expression analysis, qRT-PCR

\footnotetext{
*Correspondence: jqhuang@hainanu.edu.cn; thtiger@163.com

Hainan Key Laboratory for Sustainable Utilization of Tropical Bioresources, College of Tropical Crops, Hainan University, No.58 Renmin Avenue, Haikou 570228, Hainan, People's Republic of China
}

(c) The Author(s). 2020 Open Access This article is licensed under a Creative Commons Attribution 4.0 International License, which permits use, sharing, adaptation, distribution and reproduction in any medium or format, as long as you give appropriate credit to the original author(s) and the source, provide a link to the Creative Commons licence, and indicate if changes were made. The images or other third party material in this article are included in the article's Creative Commons licence, unless indicated otherwise in a credit line to the material. If material is not included in the article's Creative Commons licence and your intended use is not permitted by statutory regulation or exceeds the permitted use, you will need to obtain permission directly from the copyright holder. To view a copy of this licence, visit http://creativecommons.org/licenses/by/4.0/ The Creative Commons Public Domain Dedication waiver (http://creativecommons.org/publicdomain/zero/1.0/) applies to the data made available in this article, unless otherwise stated in a credit line to the data. 


\section{Background}

Plants suffer from various biotic and abiotic stresses during growth and development, and one of the most important stresses is disease caused by pathogens. Once attacked by a pathogen, plants perceive and recognize the pathogen/microbe-associated molecular patterns (PAMPs/MAMPs) via cell surface receptors and trigger an immune response, which is known as PAMP or MAMP-triggered immunity (PTI/MTI) [1]. In pathogenic microorganism, flagellin (flg22), elongation factor $\mathrm{Tu}$ (EF-Tu), peptidoglycan (PGN) and lipopolysaccharide (LPS) from bacterial, with chitin, chitosan from fungal and $\beta$-glucans from oomycetes are typical PAMPs [2]. Once attacked by pathogens, plant first to activate defenses by plant pattern recognition receptors (PRRs) which located in cell surface. Surface-localized receptor kinases (RKs), especially the TM-LRR are the most known PRR proteins. One kind of the RKs is surfacelocalized receptor and the other are receptor-like proteins. These proteins can detect conserved PAMPs by various ligand-binding ectodomains to activate plant PTI/MTI [3]. Compared with PRR-RKs, the PRR-RLPs proteins have the same overall structures but lack an intracellular kinase domain.

In plants, there are two kinds of transmembrane receptor-like kinases (PRR-RLKs) are well-known. One kind of PRR-RKs are receptor-like serine/threonine kinases and the other are receptor histidine kinases. The RLKs are a large family of well-studied kinases with an extracellular domain, a transmembrane domain and an intracellular kinase domain in generally [4]. RLKs are sub-classed to leucine-rich repeat (LRR), lysine motifs receptor kinase (LYK) and Catharanthus roseus RLK1like (CrRLK1L) according to its N-terminal extracellular domain and the leucine-rich repeat receptor like kinases (LRR-RLKs) were the best studied [5]. RLKs are cell surface localized and can recognize distinct ligands of microbial origin. For example, the flg22 and EF-Tu elf18 are detected by flagellin sensitive 2 (FLS2) and elongation factor $\mathrm{Tu}$ receptor (EFR) respectively which belong to LRR-RLKs. The chitin elicitor receptor kinases (CERK1) through homodimerization and phosphorylation mediate chitin-induced signaling [6]. The LysMRK lysine motif receptor kinase5 (LYK5) binds fungal chitin to form a chitin inducible complex with CERK1 to induce plant immunity in Arabidopsis [7].

Not all kinases have extracellular or transmembrane domains, such as receptor-like cytoplasmic protein kinases (RLCKs). But these kinases also have catalytic domain in intracellular localization similar to RLKs [4]. Botrytis-induced kinase1 (BIK1), a plasma membranelocalized RLCK which defined as a serine/threonine kinase interact with FLS2 and brassinosteroid (BR) insensitive 1-associated kinase 1 (BAK1) to initiate plant immune responses to bacterial flagellin [8]. Another RLCK which named BR-signaling kinase 1 (BSK1) played as a substrate of the brassinosteroid receptor BR insensitive 1 (BRI1) to physically associates with the PAMP receptor FLS2 and positively regulates the PTI [9]. These results investigated that PRRs including receptor kinases (RKs), receptor-like proteins (RLPs) and their interacted kinases or proteins play important roles in plant defense of PTI process.

If PAMP-triggered immunity response is considerable, intracellular immunity is activated. Resistance ( $R$ ) proteins in plants directly or indirectly recognize pathogen effectors and activate effector-triggered intracellular immunity (ETI). In plants, there is a large leucine-rich repeat (LRR) family of $\mathrm{R}$ proteins with the consensus amino acid (aa) sequence motif LxxLxLxxN/CxL (x denotes any aa) [10]. Most LRRs are immune receptors and mainly participate in growth, development, the ETI process of plant-pathogen interactions, and stress defense [11]. During ETI process, secreted virulence effectors are perceived by plants intracellular immune receptors. Among those immune receptors, nucleotide binding site leucine repeat receptors (NBS-LRRs) take up a large part [3]. The NBS-LRR type proteins are classified to toll/interleukin receptor (TIR)-NBS-LRR (TNL) and coiled-coil (CC)-NBS-LRR (CNL), and these are the majority resistance $(R)$ proteins in response to pathogen infection in plants $[12,13]$.

The LRR family genes are mainly referenced to encode proteins with a leucine-rich repeat (LRR) conserved domain which including leucine-rich repeat receptor/receptor-like kinases (LRR-RKs/RLKs) subfamily, leucinerich repeat receptor/receptor-like proteins (LRR-RPs/ LRR-RLPs) subfamily, F-box/LRR-repeat protein (FBXL) subfamily and nucleotide binding site leucine rich repeat (NBS-LRR) subfamily that generally participated in plants immunity. In the aspect of structure, the main features of the LRR genes are their structural domain of 20-30 aa and their transmembrane domain containing 2-42 LRRs [14]. Previous studies showed that the LRR domain provides a versatile structural framework for protein-protein interactions and allows proteins to act as molecular switches in relation to pathogen recognition and defense activation $[15,16]$.

A typical LRR-RLK contains a N-terminal extracellular receptor domain, a single-pass transmembrane domain and a C-terminal intracellular kinase domain. The $\mathrm{N}$ terminal domain perceives signals, the single-pass domain anchors the protein within the membrane and the C-terminal kinase domain transduces signals downstream via autophosphorylation [17]. In Arabidopsis thaliana, the LRR transmembrane receptor kinase FLS2 is a pattern-recognition receptor that determines the specificity of flagellin 22 (flg22) perception [18]. A 
Capsicum annuum (pepper) LRR protein (CaLRR1) was reported to regulate plant cell death and defense by interacting with two pathogenesis-related proteins (CaPR10 and CaPR4b) and hypersensitive induced reaction 1 protein (CaHIR1) [19]. Another C. annuum plasma membrane LRR protein (CaLRR51) acts as a positive regulator in response to bacterial Ralstonia solanacearum infection [20].

In the LRR-RLK family, the LRR receptor-like serine/ threonine-protein kinase (LRR-STK) subfamily is the largest subfamily. The members play crucial roles in pathogen recognition signaling, the subsequent activation of plant defense mechanisms, and developmental control [21]. BRI1 and BAK1 are a pair of RLKs involved in BR signaling, which regulates plant growth, development, and stress responses $[22,23]$. BAK1 is also an LRR-STK that acts as a signaling regulator by interacting with BRI1 in vitro and in vivo in Arabidopsis [22]. In contrast to LRR-RLKs, LRR-RPs lack a cytoplasmic kinase domain for initiation of downstream signal transduction in plants [24].

The FBXL subfamily is widely found in animals and plants, and there are approximately $40-50$ aa in the Nterminus of the conserved F-box domain [25]. It was reported that F-box proteins are the substrate-recognition subunits of Skp1-Cullin-F-box protein (SCF) ubiquitin ligase complexes [26]. Genome-wide analyses showed that F-box genes respond to salt stress, heavy metals, and drought in soybean and the legume Medicago truncatula $[27,28]$. Auxin-signaling F-box protein 4 (AFB4), which is similar to the auxin receptor known as Toll/ interleukin-1 receptor (TIR1), plays a pivotal role in plant growth, development, and innate immunity, as shown by a combination of physiological, molecular, and genetic approaches [29]. An interesting study by Angot et.al. (2006) showed that seven type III secretion system (T3SS) effectors that contain both an LRR domain and an F-box domain promote disease in several host plants. The authors stated that this may be because they hijack their host SCF-type E3 ubiquitin ligases to interfere with the host ubiquitin/proteasome pathway to promote disease [30].

In mammals, FBXL2 was reported to interact with the pool of p85 beta subunits to control the phosphatidylinositol-3-kinase (PI3K) signaling cascade by harnessing the proteasomal degradation process [26]. Immunoprecipitation and tandem mass spectrometry (IP-MS) analysis showed that the fork head box M1 (FoxM1) transcription factor interacted with the FBXL2 protein in gastric cancer [31]. Meanwhile, Chen et al. showed that FBXL2 ubiquitinates Aurora $B$ to inhibit tumorigenesis [32]. Furthermore, research by Tosto et al. showed that FBXL7 overexpression was associated with Alzheimer's disease in a study that compared Alzheimer's disease-like transgenic mice to wild-type littermates [33]. These results show that FBXL proteins not only participate in growth, development, and disease in plants but also in mammals.

In plants, the NBS-LRR subfamily can directly associate with pathogen-derived effectors as receptors and indirectly sense effector-mediated modification of other host proteins [34]. Functioning as a molecular switch, NBS is a part of the NB-ARC ("nucleotide binding adaptor shared by APAF-1, resistance proteins, and CED-4") domain [35]. Activated plant NBS-LRRs trigger a range of immune responses, such as the hypersensitive response (HR), which culminates in death of infected cells [36]. There are two major classes of NBS-LRRs in plants: the coiled-coil motif (CC)-NBS-LRR (CNL) subfamily and the TIR-NBS-LRR (TNL) subfamily [37]. The CC-NBS-LRR subfamily have an N-terminal CC domain, while the TIR-NBS-LRR subfamily have a TIR domain [38]. A wheat CC-NBS-LRR (TaRCR1) was reported to positively contribute to the defense response to the fungal pathogen Rhizoctonia cerealis by maintaining reactive oxygen species (ROS) homoeostasis [39]. Another CCNBS-LRR protein Pm21 in wheat was proved to confer powdery mildew resistance [40]. In the plant Nicotiana benthamiana, overexpression of the novel fungal Plasmoparaviticola-induced TIR-NBS-LRR gene (VaRGA1) enhanced disease resistance and drought and salt tolerance [41]. Soybean resistance was improved to several mosaic virus strains by overexpression of soybean TIRNBS-LRR type R gene GmKR3 [42]. Similar to other LRR genes, NBS-LRR genes play important roles in plant disease defense signaling.

Pitaya (Hylocereus polyrhizus and Hylocereus undatus) is an important tropical-subtropical fruit tree found in Central America, East Asia and Southeast Asia. Canker disease caused by the fungi Neoscytalidium dimidiatum is one of the most destructive and economically important diseases of in the pitaya industry $[43,44]$. At present, due to the lack of reports on the pitaya genome, it is economic and efficient to study biotic and abiotic stress response genes using a high-throughput approach.

The fungal which named Neoscytalidium dimidiatum caused canker disease of pitaya has been isolated and identified in our previous work in Hainan Province, China [44]. Transcriptome-wide high-throughput RNASequencing (RNA-Seq) about the expression profiles of resistant genes related to Neoscytalidium dimidiatum defense in normal and diseased stem tissues were made ensue [45]. Here, we analyzed and studied the pitaya (Hylocereus polyrhizus) LRR receptor-like (HpLRR) family resistant genes in response to $N$. dimidiatum infection based on previous RNA-Seq data detail. Transcriptomics, molecular characterization, and expression 
results regarding LRR subfamilies in pitaya after $N$. dimidiatum infection have not been previously reported. This study provides an overview of the subfamily classification of LRR genes in pitaya and provides a basis for future functional studies. The evolutionary history and functions of each LRR subfamily in pitaya remains to be understood in future research.

\section{Results}

\section{Identification and phylogenetic analysis of pitaya $H p L R R$} transcriptional genes

We have obtained the differentially expressed Hiseq data induced by pitaya canker disease through RNA-seq reported in detail in our previous study [45]. In this study, a total of $272 \mathrm{HpLRR}$ transcriptional genes were identified based on previous de novo transcriptomic analysis. The heatmap analysis indicated that most LRR genes were up-regulated in diseased pitaya tissues. Among these genes, 12 (Unigene19327_All, Unigene21125_All, Unigene13635_All, CL1260.Contig2_All, CL2218.Contig1_All, Unigene19955_All, Unigene13405_All, Unigene18881_All, Unigene9087_All, Unigene13867_All, Unigene15298_All, and Unigene12636_All) were significantly up-regulated based on a log2FoldChange $(\mathrm{D} / \mathrm{N})$ $\geq 1.0$ (FDR $\leq 0.001$ ) (Supplemental Material 1). The 272 $H p L R R$ transcriptional genes underwent a BLAST analysis (Supplemental Material 1) involving six protein databases (nr/nt, SwissProt, KEGG, COG, InterPro, and $\mathrm{GO})$. Based on the BLAST analysis, these genes were annotated as belonging to the LRR-STK subfamily (135), FBXL subfamily (49), NBS-LRR subfamily (29), LRRRLK subfamily (26), plant intracellular Ras group-related LRR (PIRL) subfamily (8), LRR transmembrane protein kinase subfamily (5), brassinosteroid LRR receptor kinase subfamily (3), and other LRR genes (17) (Table 1). Heatmaps of the $272 \mathrm{HpLRR}$ gene expression levels in D1, D3, N2 and N3 samples are presented in Figs. 1 and 2. Although LRR-STK genes belong to the LRR-RLK subfamily, in order to distinguish them from common LRR-RLK genes, separate heatmaps were constructed.

Among the $272 \mathrm{HpLRR}$ genes, 39 have no CDS, based on base sequence analysis using A plasmid Editor (ApE) software (http://jorgensen.biology.utah.edu/wayned/ape/ ). In contrast, some $H p L R R$ genes had multiple CDSs. The $33 \mathrm{HpLRR}$ genes with CDSs $>1.0 \mathrm{~kb}$ were selected for gene annotation and gene structure analysis, while the four HpLRR genes with the longest CDSs were selected for further expression analysis (at different fungal infection stages, in different pitaya tissues, and after plant hormone treatment). The aa sequence and molecular weight of the $233 \mathrm{HpLRR}$ genes with CDSs varied greatly, from $30 \mathrm{aa} / 3.382 \mathrm{kDa}$ to $1138 \mathrm{aa} / 128.777 \mathrm{kDa}$ (Supplementary Material 2). The aa sequences of the $233 \mathrm{HpLRR}$ genes were used for phylogenetic analysis using MEGA 6.0 [46], and the nine significantly upregulated genes with CDSs are marked in red (Fig. 3). The phylogenetic analysis showed that the 233genes could be divided into eight subfamilies (Fig. 3 and Table 1). This indicated that these genes have some degree of aa-level similarity, suggesting evolutionary relationships. Nevertheless, the base and aa sequence evolutionary results were not consistent (Table 1), due to the degeneracy of codons.

\section{Gene structure of the $33 \mathrm{HpLRR}$ transcriptional genes with CDSs $>1.0 \mathrm{~kb}$}

It was hard to conduct further research on all the LRR genes as there were many of them. Hence, we selected genes with CDSs $>1.0 \mathrm{~kb}$ for gene structure analysis and conserved motifs analysis (Fig. 4) for further study. The detailed information of these 33 genes with CDSs $>1.0 \mathrm{~kb}$ was showed in Table 2. All $33 H p L R R$ transcriptional genes have upstream and downstream sequences. CL1599.Contig2_All and CL1599.Contig1_All; CL971. Contig1_All and CL971.Contig2_All; CL1165.Contig6_All and CL1165.Contig3_All; Unigene11462_All, Unigene 8930_All, and Unigene7537_All; and Unigene2712_All and CL332.Contig2_All have 99-100\% similarity, indicating that each set of two or three genes have close evolutionary relationships.

\section{Verification of 12 differentially expressed LRR genes (DEGs) by qRT-PCR}

Based on a PossionDis analysis, 12 genes were significantly up-regulated $(\mid \log 2$ FoldChange $(\mathrm{D} / \mathrm{N}) \mid \geq 1.0, \mathrm{FDR} \leq 0.001)$ among the total set of 272 LRR genes. Among these 12 genes, seven (Unigene15298_All, Unigene21125_All, Unigene13635_All, CL1260.Contig2_All, Unigene13867_All, Unigene19327_All, and Unigene9087_All) were annotated as belonging to the LRR-STK subfamily, three (CL2218.Contig1_All, Unigene19955_All and Unigene1 3405_All) as belonging to the FBXL subfamily, one (Unigene18881_All) as a disease resistance gene, and one (Unigene12636_All) as belonging to the PIRL subfamily.

To verify the RNA-Seq results, qRT-PCR assays were performed. The qRT-PCR expression level trends of 11 of the 12 genes (not Unigene19955_All) were consistent with the RNA-Seq results (Fig. 5). In addition, three of the 12 (Unigene15298_All, Unigene21125_All, and CL2218.Contig1_All) have no CDS, and the number of aa of the remaining nine genes ranged from 35 to 290 (Supplementary Material 2).

\section{Expression profiles of four $H p L R R$ genes under different stages of $N$. dimidiatum infection in different pitaya species}

Four $H p$ LRR genes (CL445.Contig4_All, Unigene28_All, CL28.Contig2_All, and Unigene2712_All) were selected 
Table 1 Classification of LRRs family genes based on six protein databases and phylogenetic analysis.

\begin{tabular}{|c|c|c|c|c|}
\hline \multirow{9}{*}{$\begin{array}{l}\text { Based on } \\
\text { Six Protein } \\
\text { Databases } \\
\text { (Total 272) }\end{array}$} & Classification & Number & Up regulation & Description of Classification \\
\hline & । & 135 & $\begin{array}{l}\text { Unigene19327_All, } \\
\text { Unigene21125_All, } \\
\text { Unigene13635_All, } \\
\text { Unigene9087_All, } \\
\text { Unigene13867_All, } \\
\text { CL1260.Contig2_All }\end{array}$ & LRR-STK subfamily \\
\hline & $\|$ & 26 & Unigene15298_All & LRR-RLK subfamily \\
\hline & III & 29 & --- & NBS-LRR subfamily \\
\hline & IIII & 49 & $\begin{array}{l}\text { CL2218.Contig1_All, } \\
\text { Unigene19955_All, } \\
\text { Unigene13405_All }\end{array}$ & FBXL subfamily \\
\hline & V & 8 & Unigene12636_All & PIRL subfamily \\
\hline & $\mathrm{Vl}$ & 5 & --- & LRR transmembrane protein kinase \\
\hline & VII & 3 & --- & Brassinosteroid LRR receptor kinase \\
\hline & VIII & 17 & Unigene18881_All & Other LRR genes \\
\hline \multirow{9}{*}{$\begin{array}{l}\text { Based on } \\
\text { Phylogenetic } \\
\text { Analysis } \\
\text { (Total 233) }\end{array}$} & Classification & Number & aa length & Gene annotation and number \\
\hline & । & 16 & $31-465$ & $\begin{array}{l}\text { LRR receptor-like/receptor-like STK (9); NBS-LRR (3); } \\
\text { FBXL (2); Brassinosteroid LRR receptor kinase (1); } \\
\text { LRR transmembrane protein kinase (1) }\end{array}$ \\
\hline & $\|$ & 32 & $31-927$ & $\begin{array}{l}\text { LRR receptor-like /receptor-like STK (16); FBXL (6); } \\
\text { NBS-LRR (6); LRR transmembrane kinase (1); } \\
\text { Brassinosteroid LRR receptor kinase (1); PIRL (1); } \\
\text { LRR and ubiquitin-like domain-containing protein (1) }\end{array}$ \\
\hline & III & 24 & $32-604$ & $\begin{array}{l}\text { LRR receptor-like/receptor-like STK (15); FBXL (3); } \\
\text { NBS-LRR (3); PIRL (2); Brassinosteroid LRR receptor } \\
\text { kinase-like (1) }\end{array}$ \\
\hline & IV & 30 & $31-1138$ & $\begin{array}{l}\text { LRR receptor-like/receptor-like STK (16); NBS-LRR (6); } \\
\text { FBXL (5); LRR transmembrane protein kinase (3) }\end{array}$ \\
\hline & V & 26 & $34-364$ & LRR receptor-like/receptor-like STK (24); FBXL (2) \\
\hline & $\mathrm{Vl}$ & 27 & $36-501$ & $\begin{array}{l}\text { LRR receptor-like/receptor-like STK (16); FBXL (5); } \\
\text { PIRL (4); NBS-LRR (1); Disease resistance protein } \\
\text { RGA4 (1) }\end{array}$ \\
\hline & VII & 24 & $32-515$ & $\begin{array}{l}\text { LRR receptor-like/receptor-like STK (15); FBXL (7); } \\
\text { NBS-LRR (2) }\end{array}$ \\
\hline & VIII & 54 & $30-504$ & $\begin{array}{l}\text { LRR receptor-like/receptor-like STK (36); FBXL (11); } \\
\text { NBS-LRR (5); PIRL (1); Disease resistance RPP13-like } \\
\text { protein (1) }\end{array}$ \\
\hline
\end{tabular}

FBXL F-box/LRR-repeat protein, LRR leucine-rich repeat, NBS nucleotide-binding site, PIRL plant intracellular Ras group-related LRR, RLK receptor-like kinase, STK serine/threonine-protein kinase

for the subsequent expression analysis as they had the four longest CDSs (Supplementary Material 2). The conserved domain analysis indicated that CL445.Contig4_ All and Unigene2712_All were NBS-LRR type resistance proteins, while Unigene28_All and CL28.Contig2_All were probable LRR-STKs (Fig. 6). The four genes have 3-9 LRR motifs (Fig. 6). In addition, CL445.Contig4_All and Unigene2712_All belong to the RX-CC_like family ("Coiled-coil domain of the potato virus $\mathrm{X}$ resistance protein and similar proteins") and the NB-ARC family. Unigene28_All and CL28.Contig2_All have specific hits regarding the STKc_IRAK family ("Catalytic domain of the serine/threonine kinases, interleukin-1 receptor associated kinases, and related STKs") and a common LRR-RLK (the PLN00113 superfamily). CL28.Contig2 All has a specific malectin-like domain; malectin is a novel endoplasmic reticulum carbohydrate-binding protein and a candidate player in the early steps of protein $\mathrm{N}$-glycosylation [47].

The pitaya stems presented obvious symptoms after 3 days of $N$. dimidiatum infection and rotted by day 15 (Fig. 7). However, compared with red-fleshed pitaya (Fig. 7a), the white-fleshed pitaya (Fig. 7b) possessed strong resistance. Due to the protective hard wax coat on the pitaya stems, the expression levels of the four LRR genes peaked after 3 4 days of infection in red-fleshed pitaya 


\begin{tabular}{|c|c|c|c|c|c|c|c|}
\hline D1 I & D3 & N2 & Gene ID and Descripition & D1 & D3 & N2 $\mathrm{N}$ & Gene ID and Descripition \\
\hline 2 & 1 & 0 & 0 CL340.Contig1_All LRR-STK BAM1 & 0 & 1 & 0 & 0 CL28.Contig1_All LRR-STK At1g67720 iso form X1 \\
\hline 3 & 2 & 1 & 0 CL340.Contig2_All LRR-STK BAM1 & 0 & 1 & 0 & 0 Unigene6431_All LRR-STK At1g67720-like \\
\hline 2 & 5 & 1 & 1 CL340.Contig7_All LRR-STK BAM1 & 2 & 4 & 1 & 1 CL28.Contig2_All LRR-STK At1g67720-like \\
\hline 1 & 3 & 1 & 1 Unigene8225_All LRR-STK & 1 & 0 & 0 & 0 Unigene23245_All LRR-STK At1g74360 \\
\hline 0 & 1 & 0 & 0 Unigene14565_All LRR-STK EFR & 0 & 1 & 0 & 0 Unigene17869_All LRR-STK At2g 16250 \\
\hline 0 & 1 & 0 & 0 Unigene18117_All LRR-STK EFR & 1 & 0 & 0 & 0 Unigenel0557_All LRR-STK At2g 16250 \\
\hline 1 & 0 & 0 & 0 Unigene6414_All LRR-STK EFR-like & 1 & 1 & 0 & 0 Unigene10791_All LRR-STK At2g 16250 \\
\hline 1 & 0 & 0 & 0 CL13.Contig1_All LRR-STK EFR-like & 1 & 1 & 0 & 0 Unigene4714_All LRR-STK At2g16250-like \\
\hline 1 & 0 & 0 & 0 CL13.Contig2_All LRR-STK EFR-like & 0 & 0 & 0 & 0 Unigene14537_All LRR-STK At2g24230-like \\
\hline 0 & 2 & 0 & 0 CL1417.Contig2_All LRR-STK ERECTA-like iso form X2 & 0 & 1 & 0 & 0 Unigene16909_All LRR-STK At2g24230-like \\
\hline 1 & 0 & 0 & 0 Unigene24242_All LRR-STK FEI & 0 & 1 & 1 & 0 Unigene18179_All LRR-STK At3g47570 \\
\hline 3 & 1 & 1 & 0 Unigene1372_All LRR-STK FEI & 0 & 1 & 0 & 0 Unigene19845_All LRR-STK At3g47570 \\
\hline 2 & 2 & 2 & 1 CL877.Contig2_All LRR-STK FEI 1 & 0 & 1 & 0 & 0 Unigene19994_All LRR-STK At3g47570 \\
\hline 0 & 0 & 0 & 0 Unigene15090_All LRR-STK FEI 1 & 0 & 1 & 0 & 0 Unigene13782_All LRR-STK At3g47570 \\
\hline 0 & 1 & 0 & 0 CL877.Contig4_All LRR-STK FEI 1 & 0 & 3 & 0 & 0 Unigene13635_All LRR-STK At3g47570 \\
\hline 1 & 3 & 1 & 1 CL877.Contig1_All LRR-STK FEI 1 -like & 1 & 3 & 0 & 0 CL1260.Contig2_All LRR-STK At3g47570 \\
\hline 0 & 1 & 0 & 0 CL877.Contig3_All LRR-STK FEI 1 -like isoform 1 & 0 & 3 & 0 & 0 CL986.Contig2_All LRR-STK At3g47570 \\
\hline 1 & 0 & 0 & 0 Unigene26481_All LRR-STK FLS2 & 1 & 1 & 0 & 1 Unigene5348_All LRR-STK At3g47570 \\
\hline 0 & 0 & 0 & 0 Unigene21861_All LRR-STK FLS2 & 0 & 2 & 0 & 0 CL592.Contig1_All LRR-STK At3g47570 \\
\hline 1 & 0 & 0 & 0 Unigene26736_All LRR-STK FLS2 & 3 & 2 & 14 & 9 Unigene7164_All LRR-STK At3g47570 \\
\hline 0 & 0 & 1 & 1 Unigene13319_All LRR-STK FLS2 & 0 & 1 & 0 & 0 Unigene7827_All LRR-STK At3g47570 \\
\hline 0 & 1 & 0 & 0 Unigenel 6887_All LRR-STK FLS2 & 0 & 0 & 0 & 0 Unigenel3612_All LRR-STK At3g 47570 iso form X2 \\
\hline 0 & 1 & 0 & 0 Unigene7318_All LRR-STK FLS2 & 0 & 2 & 0 & 0 Unigene19327_All LRR-STK At3g47570-like \\
\hline 0 & 1 & 1 & 0 Unigenel1169_All LRR-STK FLS2 & 1 & 0 & 0 & 0 CL1260.Contig1_All LRR-STK At3g47570-like \\
\hline 14 & & 2 & 4 Unigene8646_All LRR-STK FLS2 & 2 & 2 & 0 & 0 Unigene13867_All LRR-STK At3g47570-like \\
\hline 0 & 1 & 0 & 0 Unigene1 8552_All LRR-STK FLS2-like & 0 & 2 & 0 & 0 CL471.Contig2_All LRR-STK At3g47570-like \\
\hline 0 & 2 & 0 & 0 Unigene2003_All LRR-STK FLS2-like & 1 & 4 & 0 & 0 CL1260.Contig3_All LRR-STK At3g47570-like \\
\hline 4 & 1 & 0 & 0 Unigene5824_All LRR-STK FLS2-like & 1 & 0 & 0 & 0 Unigene26202_All LRR-STK At4g 08850 \\
\hline 0 & 0 & 0 & 0 Unigene18671_All LRR-STK GSO1 & 1 & 1 & 1 & 0 Unigene1367_All LRR-STK At4g 08850 \\
\hline 1 & 1 & 0 & 0 Unigene13266_All LRR-STK GSO1-like & 1 & 1 & 0 & 0 CL2283.Contig2_All LRR-STK At4g08850-like \\
\hline 1 & 2 & 0 & 0 Unigene9087_All LRR-STK GSO1-like & 0 & 2 & 0 & 0 Unigene13618_All LRR-STK At4g20940 \\
\hline 0 & 1 & 0 & 0 Unigene20408_All LRR-STK GSO2 & 1 & 0 & 0 & 0 Unigene24779_All LRR-STK At4g36180 \\
\hline 0 & 1 & 0 & 0 Unigene2 1250_All LRR-STK GSO2 iso form X2 & 1 & 0 & 0 & 0 Unigene25652_All LRR-STK At4g36180 \\
\hline 2 & 4 & 0 & 0 CL1426.Contig3_All LRR-STK GSO2-like isoform X7 & 0 & 2 & 0 & 1 Unigene14631_All LRR-STK At4g36180 \\
\hline 1 & 1 & 0 & 0 CL649.Contig6_All LRR-STK HSL2 & 0 & 0 & 0 & 1 Unigene14344_All LRR-STK At4g 36180 \\
\hline 0 & 2 & 0 & 0 Unigene21125_All LRR-STK RCH1 & 0 & 1 & 0 & 0 Unigene13989_All LRR-STK At4g36180 \\
\hline 0 & 2 & 1 & 0 Unigene9429_All LRR-STK RCH1 & 1 & 2 & 0 & 0 CL1426.Contig2_All LRR-STK At4g36180 \\
\hline 1 & 0 & 0 & 0 Unigene3969_All LRR-STK RCH1 & 0 & 1 & 1 & 1 Unigene8268_All LRR-STK At4g36180 \\
\hline 1 & 1 & 0 & 0 Unigene12595_All LRR-STK RPK2-like & 0 & 1 & 0 & 0 Unigene21504_All LRR-STK At4g36180 iso form X2 \\
\hline 0 & 3 & 0 & 0 Unigene19708_All LRR-STK & 0 & 1 & 0 & 0 CL1510.Contig1_All LRR-STK At4g36180-like \\
\hline 9 & & 12 & 7 Unigene1 812_All LRR-STK & 0 & 0 & 0 & 0 CL1510.Contig2_All LRR-STK At4g36180-like \\
\hline 0 & 1 & 0 & 0 Unigene19023_All LRR-STK At1g67720 & 1 & 1 & 0 & 0 CL1510.Contig3_All LRR-STK At4g36180-like \\
\hline 0 & 1 & 0 & 0 Unigene19111_All LRR-STK At1g67720 & 1 & 3 & 0 & 0 Unigenel 1953_All LRR-STK At5g 10290 iso form X2 \\
\hline 2 & 1 & 0 & 0 CL1168.Contig3_All LRR-STK At1g67720 & 1 & 3 & 1 & 1 CL1 165.Contig5_All LRR-STK At5g10290-like isoform X1 \\
\hline 1 & 1 & 0 & 0 Unigene3068_All LRR-STK & 0 & 0 & 0 & 0 CL1 165.Contig6_All LRR-STK At5g10290-like isoform X1 \\
\hline 0 & 3 & 1 & 1 Unigene9555_All LRR-STK At1g06840-like & 1 & 3 & 0 & 0 CL1 165.Contig2_All LRR-STK At5g10290-like isoform X1 \\
\hline 0 & 0 & 1 & 0 Unigene15766_All LRR-STK Atlg12460 & 0 & 2 & 0 & 0 CL1 165.Contig3_All LRR-STK At5g10290-like iso form X1 \\
\hline 0 & 1 & 0 & 0 Unigene20363_All LRR-STK Atlg 14390 & 1 & 0 & 0 & 0 CL1 165.Contig4_All LRR-STK At5g 10290-like iso form X1 \\
\hline 0 & 1 & 0 & 0 Unigene7662_All LRR-STK At1 g 14390 & 2 & 6 & 2 & 1 CL1 165.Contig1_All LRR-STK At5g 10290-like isoform X1 \\
\hline 1 & 3 & 1 & 1 Unigene179_All LRR-STK At1g14390 & 0 & 1 & 1 & 0 Unigene12018_All LRR-STK At5g45840-like \\
\hline 2 & 3 & 0 & 0 CL986.Contig1_All LRR-STK At1g34110 & 0 & 1 & 0 & 0 CL971.Contig1_All LRR-STK At5g45840-like \\
\hline 1 & 0 & 0 & 0 Unigene24276_All LRR-STK At1g34110 & 0 & 1 & 1 & 0 CL971.Contig2_All LRR-STK At5g45840-like \\
\hline 0 & 1 & 0 & 0 Unigene15670_All LRR-STK At1g34110 & 1 & 3 & 1 & 0 Unigene6823_All LRR-STK At5g45840-like \\
\hline 0 & 1 & 0 & 0 Unigene17878_All LRR-STK At1g51860 & 1 & 0 & 0 & 0 Unigene29_All LRR-STK RFK1 \\
\hline 1 & 0 & 1 & 0 Unigene2355_All LRR-STK At1g51880-like & 4 & 3 & 1 & 1 Unigene28_All LRR-STK RFK1 isoform X1 \\
\hline 2 & 1 & 0 & 0 Unigene7537_All LRR-STK At1 g 53430 & 1 & 1 & 0 & 0 Unigene7824_All LRR-STK RKF3 \\
\hline 1 & 0 & 0 & 0 Unigene9123_All LRR-STK Atlg53430-like & 1 & 1 & 0 & 0 Unigene799_All LRR-STK RKF3 \\
\hline 1 & 1 & 0 & 0 Unigene8034_All LRR-STK At1g53430-like & 2 & 2 & 1 & 1 Unigene705_All LRR-STK RPK1 \\
\hline 1 & 1 & 0 & 0 Unigene362_All LRR-STK At1 g53430-like & 0 & 1 & 0 & 0 CL592.Contig2_All LRR-STK \\
\hline 1 & 1 & 0 & 0 CL6.Contig2_All LRR-STK At1g53430-like & 3 & 2 & 0 & 0 Unigene8930_All LRR-STK At1g56130-like \\
\hline 0 & 1 & 0 & 0 Unigene20264_All LRR-STK At1 g53440-like & 1 & 1 & 0 & 0 Unigene308_All LRR-STK At1g56140 \\
\hline 1 & 0 & 0 & 0 Unigene1275_All LRR-STK At1g53440-like & 2 & 2 & 0 & 0 Unigene1 1462_All LRR-STK At1g 56140 \\
\hline 1 & 1 & 0 & 0 Unigene3580_All LRR-STK Atlg53440-like & 0 & 2 & 0 & 0 Unigene15793_All LRR-STK At1g63430 \\
\hline 1 & 0 & 0 & 0 Unigene26043_All Probable LRR-STK Atlg56130 & 0 & 1 & 0 & 0 CL2032.Contig2_All LRR-STK At1 g63430 isoform X3 \\
\hline 2 & 0 & 0 & 0 CL10.Contig1_All Probable LRR-STK At1g56130 & 1 & 0 & 0 & 0 Unigene26099_All LRR-STK At1g06840 \\
\hline 0 & 1 & 0 & 0 Unigene21553_All Probable LRR-STK At1 g56130-like & 0 & 0 & 0 & 0 CL1240.Contig1_All LRR-STK At1g06840 \\
\hline 0 & 1 & 0 & 0 Unigene17845_All Probable LRR-STK At1g56130-like & 0 & & 0 & 0 CL1240.Contig2_All LRR-STK At1g06840 iso form X3 \\
\hline 1 & 1 & & & & & & \\
\hline
\end{tabular}

Fig. 1 Expression profiles of LRR-STK genes in four samples. N2: normal sample 2; N3: normal sample 3; D1: diseased sample 1; D3: diseased sample 3. LRR-STK: leucine-rich repeat receptor-like serine/threonine protein kinase

while 4 6 days in white-fleshed pitaya. The expression results (Fig. 8a) suggested that, in red fleshed pitaya (Hylocereus polyrhizus), compared with control level (0 h), CL445.Contig4_All expression first decreased and then suddenly increased on day 3 , then decreased, and finally exhibited an increasing trend but remained lower than the control level. Unigene28_All and CL28.Contig2_All expression levels were increased after $N$. dimidiatum infection, but Unigene28_All reached a peak on day 3 while CL28.Contig2_All reached a peak on day 4. In contrast to the other three genes, Unigene2712_All expression always decreased compared with the control level. These results indicated that the four genes participated in the fungal infection response of pitaya, especially CL28.Contig2_All, which was significantly upregulated on day 4 . In contrast to the other three genes, 


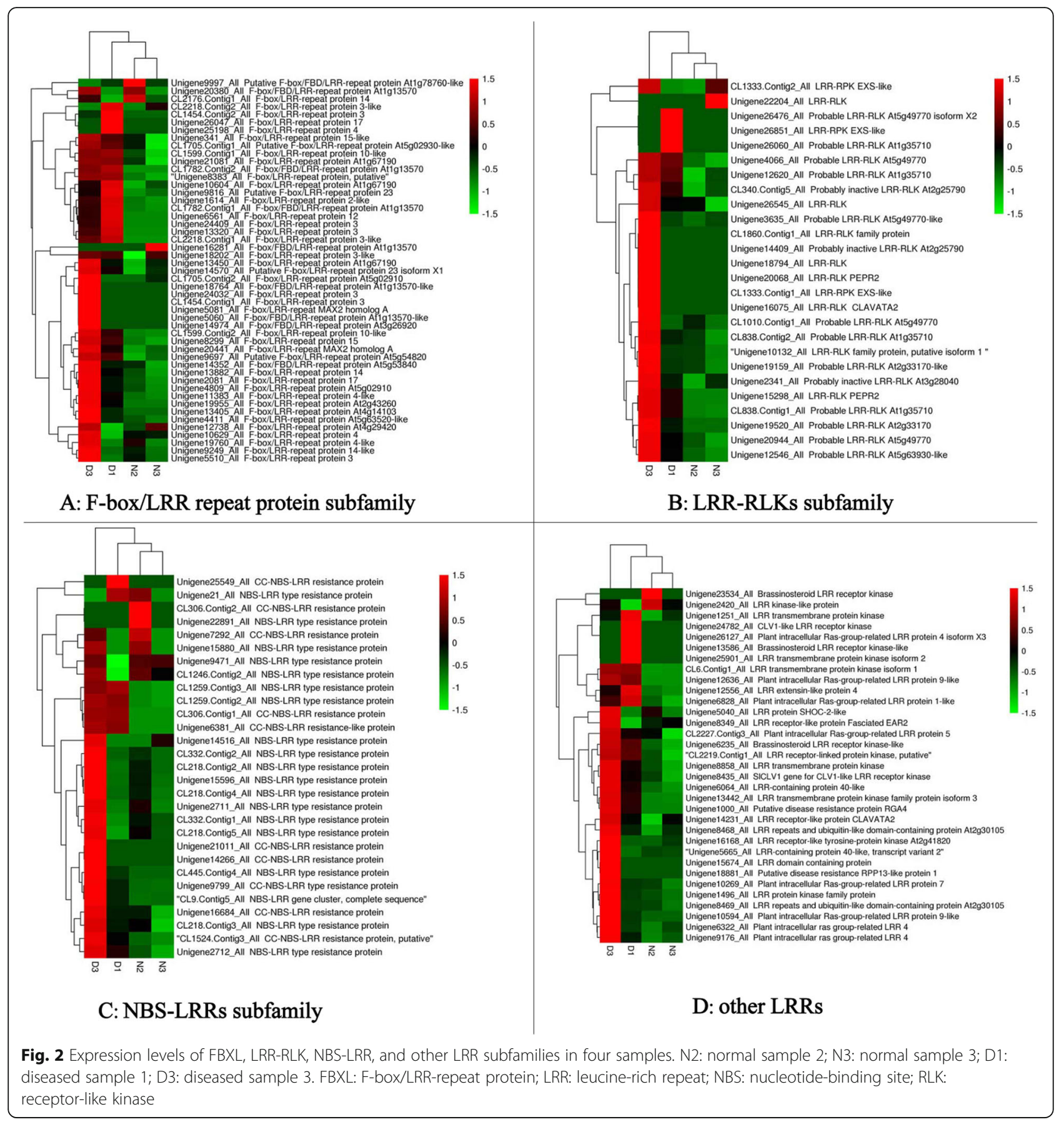

Unigene2712_All may act as a negative regulator in plant-pathogen interactions. In the white-fleshed pitaya (Hylocereus undatus), the genes expression has the similarity trend. However, the infection time of the genes reached a peak in white-fleshed pitaya was later 1 day compared with red-fleshed pitaya (Fig. 8b). The four $H p L R R$ genes expression files results were consistent with the infection symptoms of the two different pitaya species (Fig. 7).
Tissue-specific expression profiles of the four HpLRR genes

Tissue-specific genes (also known as luxury genes) are genes whose products have specific functions in specific cell types. To investigate whether the four $H p$ LRR genes we selected have specific functions in specific cells, tissue-specific expression profiles were obtained by qRTPCR for 14 pitaya tissues (Fig. 9). CL445.Contig4_All was mainly expressed in the pericarp of a young green 


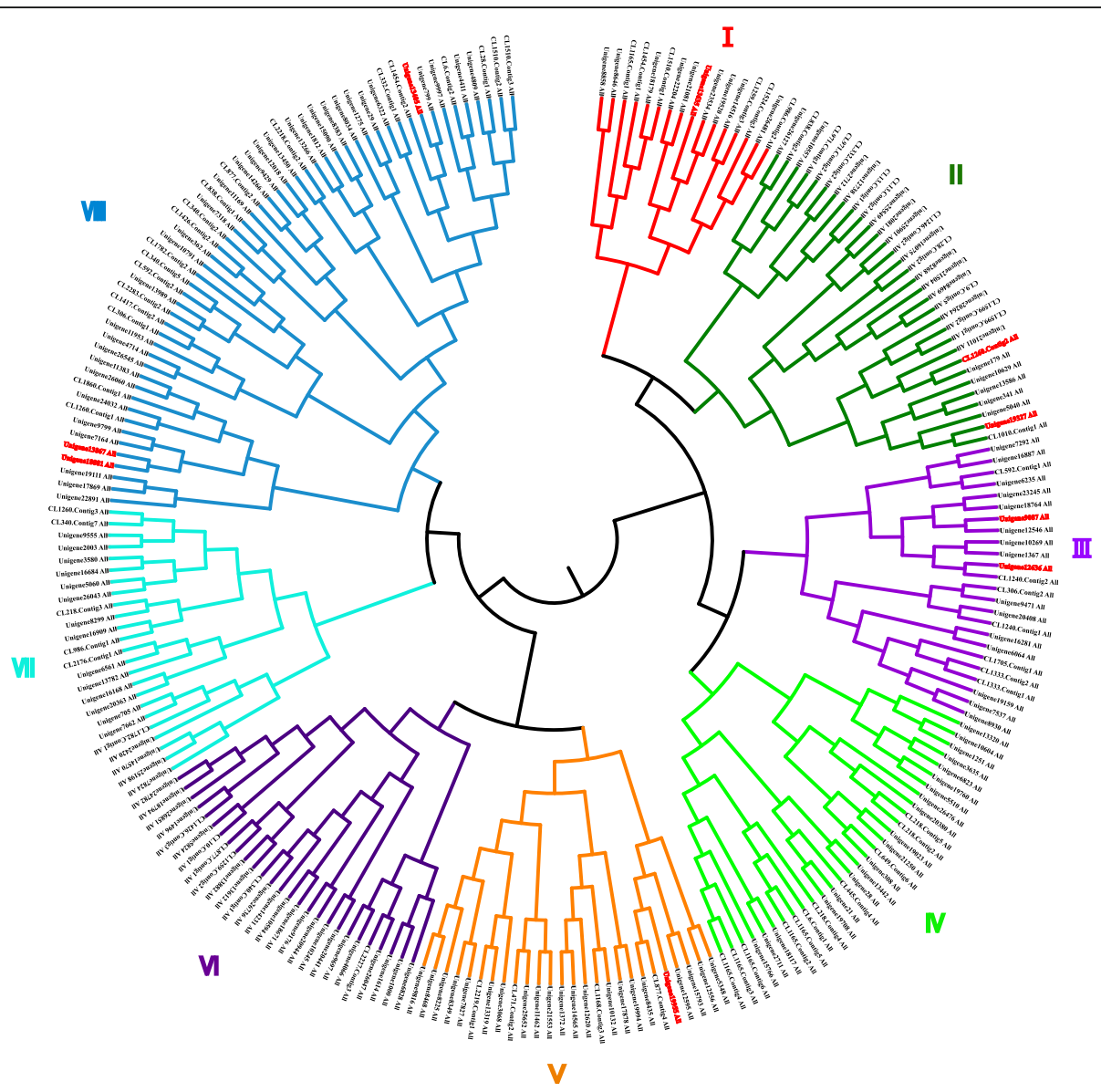

Fig. 3 Phylogenetic analysis of 233 HpLRR genes with coding sequences (CDSs) divided to eight subgroups. The 9 red genes are LRR genes that were significantly up-regulated

fruit; Unigene28_All was mainly expressed in the stamen, petal, and fruit pulp of both a young green fruit and a red fruit; CL28.Contig2_All was significantly expressed in the pericarp of both a young green fruit and a red fruit, and Unigene2712_All was mainly expressed in the flower bud. These results showed that Unigene28_All was significantly up-regulated and may play pivotal roles in pitaya flower and fruit growth and development.

\section{Expression profiles of the four $H p L R R$ genes in response to $S A, A B A$, and MeJA treatments}

In plants, hormones play important roles in response to a wide range of biotic and abiotic stress signaling networks. SA, ABA, jasmonates (JAs), and ethylene have crucial well-known roles in plant disease and pest resistance [48]. To better understand the four HpLRR genes' responses to hormonal regulation of the plant-pathology interaction pathways, the expression patterns of these genes in response to $\mathrm{SA}, \mathrm{ABA}$, and MeJA treatments were assessed by qRT-PCR (Fig. 10). All four genes responded to the three hormones to some degree.
Unigene28_All expression was significantly changed after $2 \mathrm{~h}$ of ABA treatment. CL28.Contig2_All was prominently expressed at $48 \mathrm{~h}$ of SA treatment. Unigene2712_All was significantly down-regulated by ABA, but significantly up-regulated by SA, reaching a peak at $24 \mathrm{~h}$. The results showed that Unigene28_All and CL28.Contig2_All may play pivotal roles in the hormone-mediated disease resistance response.

\section{Discussion}

In the present study, we identified 272 LRR genes in pitaya in the de novo transcriptome assembly analysis, and 233 of them have CDSs. Most of these genes belonged to the LRR-RLK, NBS-LRR, or FBXL subfamilies and, within the LRR-RLK subfamily, the LRR-STK subfamily had the largest number of genes. The results of annotation and conserved domain analysis showed that LRR genes have the typical LxxLxLxxNxL domain and most of which were participated in plant disease. Despite having distinct evolutionary origins, most animals and plants need cell-surface or intracellular immune receptors with an LRR domain to detect 

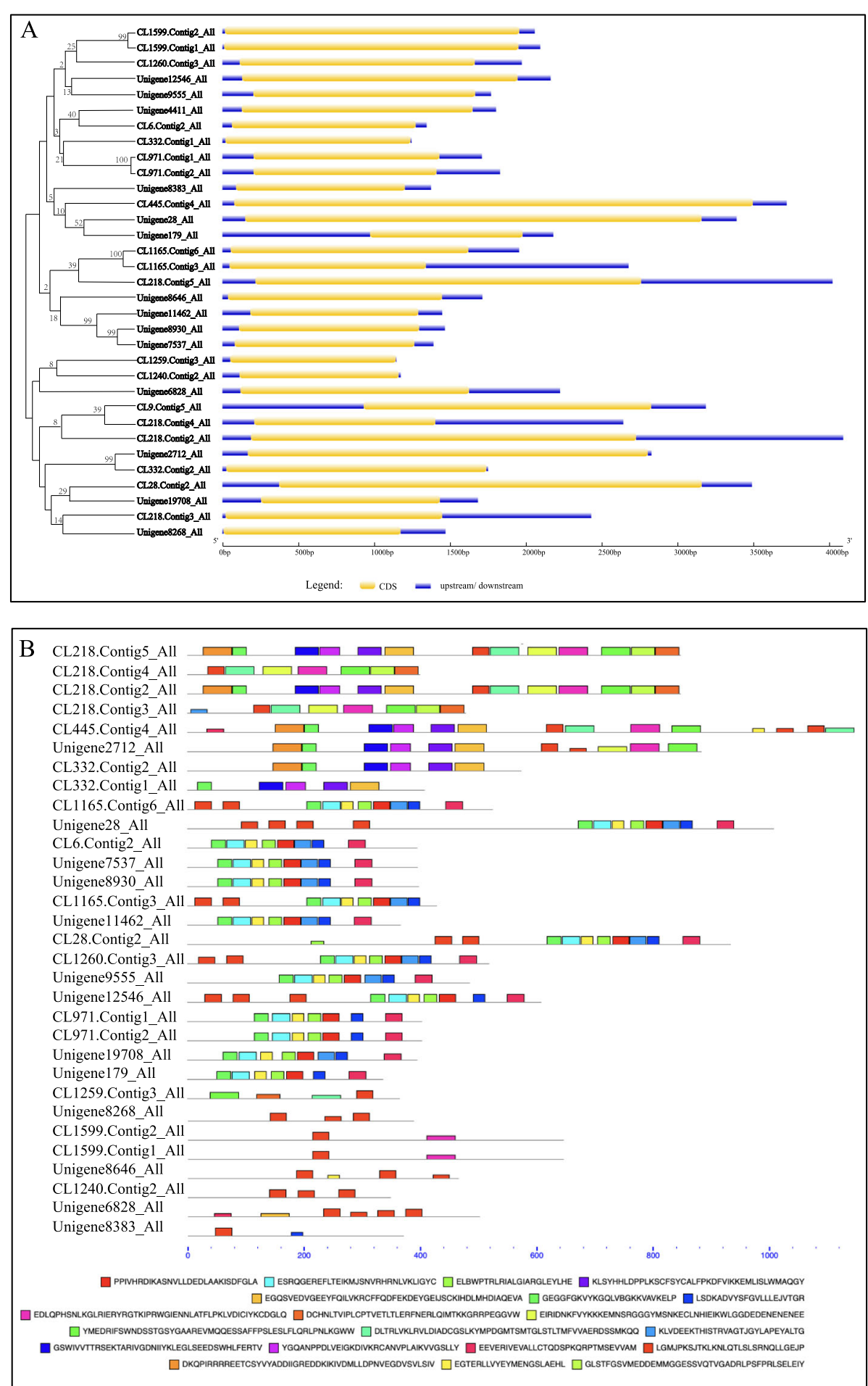

Fig. 4 Gene structure and conserved motifs analysis of the 33 pitaya HpLRR transcriptional genes with coding sequences (CDSs) > 1.0 kb. a Gene structures: Yellow represents the gene CDS and blue represents the upstream or downstream gene sequences. $\mathbf{b}$ Conserved motifs of the CDS. The conserved motifs were using the MEME program. Different motifs were highlighted with different color boxes

pathogens and trigger defense responses [49]. In mammals, LRR kinases play key roles in disease, especially Parkinson's and Crohn's diseases [50, 51]. In plants, most of these LRRs are resistance proteins and can directly associate with pathogen-derived effectors or indirectly sense effector-mediated modification of other host 
Table 2 Information of the 33 pitaya HpLRR transcriptional genes with CDSs $>1.0 \mathrm{~kb}$

\begin{tabular}{|c|c|c|c|c|c|c|}
\hline Unigene & Length & CDS & N-FPKM & D -FPKM & log2FoldChange(D/N) & Annotation (nr, SwissProt, KEGG, COG, InterPro, GO) \\
\hline CL445.Contig4_All & 3716 & 3417 & 0.49 & 1.77 & 1.85 & NBS-LRR type resistance protein \\
\hline Unigene28_All & 3386 & 3006 & 0.88 & 3.82 & 2.13 & Probable LRR receptor-like STK RFK1 isoform X1 \\
\hline CL28.Contig2_All & 3487 & 2784 & 0.65 & 2.89 & 2.15 & Probable LRR receptor-like STK At1g67720-like \\
\hline Unigene2712_All & 2825 & 2636 & 1.39 & 3.10 & 1.15 & NBS-LRR type resistance protein \\
\hline CL218.Contig5_All & 4019 & 2529 & 0.62 & 0.82 & 0.42 & NBS-LRR type resistance protein \\
\hline CL218.Contig2_All & 4089 & 2529 & 0.78 & 1.41 & 0.85 & NBS-LRR type resistance protein \\
\hline CL1599.Contig2_All & 2057 & 1938 & 0.81 & 1.82 & 1.17 & FBXL 10-like \\
\hline CL1599.Contig1_All & 2093 & 1938 & 0.26 & 0.64 & 1.29 & FBXL 10-like \\
\hline Unigene12546_All & 2161 & 1815 & 0.47 & 2.1 & 2.16 & Probable LRR receptor-like protein kinase At5g63930-like \\
\hline CL332.Contig2_All & 1749 & 1714 & 1.05 & 2.34 & 1.16 & NBS-LRR type resistance protein \\
\hline CL1165.Contig6_All & 1953 & 1566 & 0.12 & 0.12 & 0.00 & Probable LRR receptor-like STK At5g10290-like isoform X1 \\
\hline CL1260.Contig3_All & 1971 & 1548 & 0.09 & 2.45 & 4.76 & Probable LRR receptor-like STK At3g47570-like \\
\hline Unigene4411_All & 1802 & 1515 & 0.23 & 1.15 & 2.35 & FBXL At5g63520-like \\
\hline Unigene6828_All & 2223 & 1506 & 0.79 & 1.7 & 1.11 & PIRL 1-like \\
\hline Unigene9555_All & 1769 & 1449 & 0.87 & 1.56 & 0.85 & Probable LRR receptor-like STK At1g06840-like \\
\hline CL218.Contig3_All & 2429 & 1428 & 0.48 & 0.64 & 0.43 & NBS-LRR type resistance protein \\
\hline Unigene8646_All & 1712 & 1398 & 3.05 & 22.85 & 2.91 & LRR receptor-like STK FLS2 \\
\hline CL9.Contig5_All & 3183 & 1389 & 1.18 & 4.88 & 2.05 & NBS-LRR gene cluster, complete sequence \\
\hline CL1165.Contig3_All & 2675 & 1281 & 0.33 & 1.15 & 1.80 & Probable LRR receptor-like STK At5g10290-like isoform X1 \\
\hline CL332.Contig1_All & 1245 & 1219 & 0.96 & 1.33 & 0.48 & NBS-LRR type resistance protein \\
\hline CL971.Contig1_All & 1708 & 1203 & 0.05 & 0.71 & 3.97 & Probable LRR receptor-like STK At5g45840-like \\
\hline CL971.Contig2_All & 1828 & 1203 & 0.48 & 0.50 & 0.04 & Probable LRR receptor-like STK At5g45840-like \\
\hline CL218.Contig4_All & 2640 & 1194 & 0.82 & 1.70 & 1.05 & NBS-LRR type resistance protein \\
\hline Unigene8930_All & 1465 & 1188 & 0.26 & 2.41 & 3.24 & Probable LRR receptor-like STK At1g56130-like \\
\hline Unigene7537_All & 1389 & 1182 & 0.22 & 1.84 & 3.09 & Probable LRR receptor-like STK At1g53430 \\
\hline CL6.Contig2_All & 1344 & 1179 & 0.11 & 0.86 & 2.96 & Probable LRR receptor-like STK At1g53430-like \\
\hline Unigene19708_All & 1682 & 1179 & 0.05 & 1.78 & 5.15 & Putative LRR receptor-like STK \\
\hline Unigene8268_All & 1469 & 1167 & 0.74 & 0.76 & 0.04 & Probable LRR receptor-like STK At4g36180 \\
\hline Unigene8383_All & 1373 & 1113 & 0.25 & 1.03 & 2.07 & Putative FBXL \\
\hline Unigene11462_All & 1447 & 1095 & 0.26 & 1.89 & 2.86 & Probable LRR receptor-like STK At1g56140 \\
\hline CL1259.Contig3_All & 1145 & 1091 & 1.09 & 4.54 & 2.06 & NBS-LRR type resistance protein \\
\hline CL1240.Contig2_All & 1174 & 1049 & 0.10 & 0.68 & 2.75 & Probable LRR receptor-like STK At1g06840 isoform X3 \\
\hline Unigene179_All & 2179 & 1005 & 1.16 & 1.79 & 0.63 & Probable LRR receptor-like STK At1g14390 \\
\hline
\end{tabular}

$N$-FPKM Fragments per kilobase of transcript per million mapped reads (FPKM) of normal samples (N); $D$-FPKM Fragments per kilobase of. transcript per million mapped reads (FPKM) of disease samples (D). FBXL F-box/LRR-repeat protein; LRR: leucine-rich repeat; NBS: nucleotide-binding site; PIRL Plant intracellular Ras group-related LRR; RLK Receptor-like kinase; STK Serine/threonine-protein kinase

proteins [52]. Diverse classes of cell-surface immune LRR receptors share important features for the initiation of resistance. The LRR genes identified in this study may play crucial roles in the pitaya immune response. The phylogenetic analysis showed that the base and aa sequence results were not consistent (Table 1) due to the degeneracy of codons. Additionally, genes with similar base sequences do not necessarily have similar functions.

Besides the three large LRR subfamilies, we also found eight PIRL genes, five LRR transmembrane protein kinase genes, three brassinosteroid related LRR receptor kinase genes, and 17 other LRR genes, such as genes for CLAVATA (CLV)-like LRR receptor kinases, the LRR protein SHOC-2-like, and the LRR receptor-like protein FASCIATED EAR2 (Fig. 2). PIRLs are a plant-specific class of LRR proteins that take part in developmental cell signaling and gene regulation, for example, in $A$. thaliana, PIRL1 and PIRL9 have redundant roles that are essential at a key transition point early in pollen development [53]. 


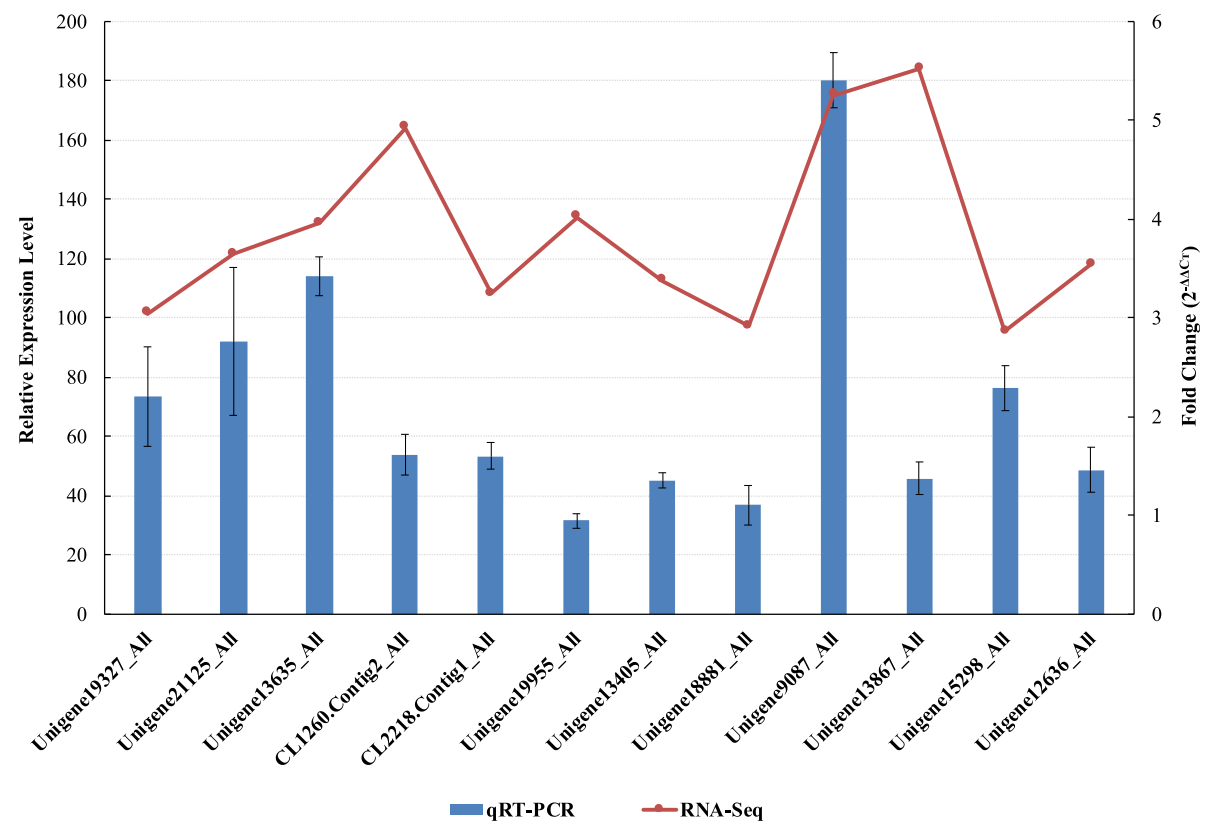

Fig. 5 Verification of 12 differentially expressed LRR genes (DEGs) by qRT-PCR assays. Gene expression levels were measured by qRT-PCR and compared with RNA-Seq results. The histogram represents the fold changes of genes (diseased [D]/normal [N]) according to qRT-PCR, and the line chart represents gene expression according to RNA-Seq. All genes selected for qRT-PCR analysis were analyzed using three biological replicates. Error bars represent $\pm \mathrm{SD}\left(2^{-\Delta \Delta C T}\right)$ based on three experiments

Most LRR-RLKs are transmembrane kinase and phosphorylate serine/threonine residues (also known as LRRSTK), and most LRR-RLKs is recognition of an extracellular ligand, which leads to activation of the intracellular kinase domain and subsequent transduction of downstream signaling pathways [54]. The five LRR transmembrane protein kinases genes that we identified may belong to the LRR-RLKs subfamily.

It has been reported that the LRR-RLK gene BAK1/ SERK3 is involved in brassinosteroid signaling and is a major modulator of PAMP-triggered immunity in $A$. thaliana and N. benthamiana [55]. The three brassinosteroid LRR receptor kinase genes in this study may play critical roles in the brassinosteroid signaling pathway in response to $N$. dimidiatum infection of pitaya.

It has been reported that CLV1 is an RLK composed of an LRR-containing extracellular domain (with putative receptor function) and a cytoplasmic STK domain (linked via a transmembrane domain); it is thought to play an important role in meristem maintenance and flower development $[54,56]$. Compared with CLV1, CLV2 is structurally similar but with a very short predicted cytoplasmic tail [57]. CLV3 is a stem cell-specific protein that activates the Arabidopsis CLAVATA stem cell signaling pathway [58]. These reports indicate that CLV proteins mainly participate in the growth and development of plants.

Four genes (CL445.Contig4_All, Unigene28_All, CL28.Contig2_All, and Unigene2712_All) were selected (because they had the four longest CDSs) for a further expression analysis. Among them, CL445.Contig4_All and Unigene2712_All were NBS-LRR resistance proteins, while Unigene28_All and CL28.Contig2_All were probable LRR receptor-like STK genes (Fig. 6). CL28.Contig2_All was significantly up-regulated on day 4 after $N$. dimidiatum infection while Unigene2712_All was significantly down-regulated in all infection stages. However, the time of the gene expression reached a peak in white-fleshed pitaya was later 1 day than that of red-fleshed pitaya (Fig. 8b). These two genes may play positive or negative roles in plant disease responses. Unigene28_All was significantly expressed on day 3 after $N$. dimidiatum infection and, based on the tissuespecific expression profiles, also significantly expressed in the stamen, petal, and fruit pulp of both a young green fruit and a red fruit. These results showed that Unigene28_All not only participates in pitaya flower and fruit growth and development but also in the disease resistance response. In addition, the expression levels of the four LRR genes peaked 3 4 days post infection in red-fleshed pitaya while $4 \sim 6$ days in whitefleshed pitaya, suggested that it probably displayed different resistance among different pitaya species.

Plant hormones, which include SA, JAs, ABA, brassinosteroid, ethylene, and auxin, act as signals to trigger and mediate plant immune responses [59]. In plants, SA is a secondary metabolite with a central role as a signaling molecule in the activation of plant 


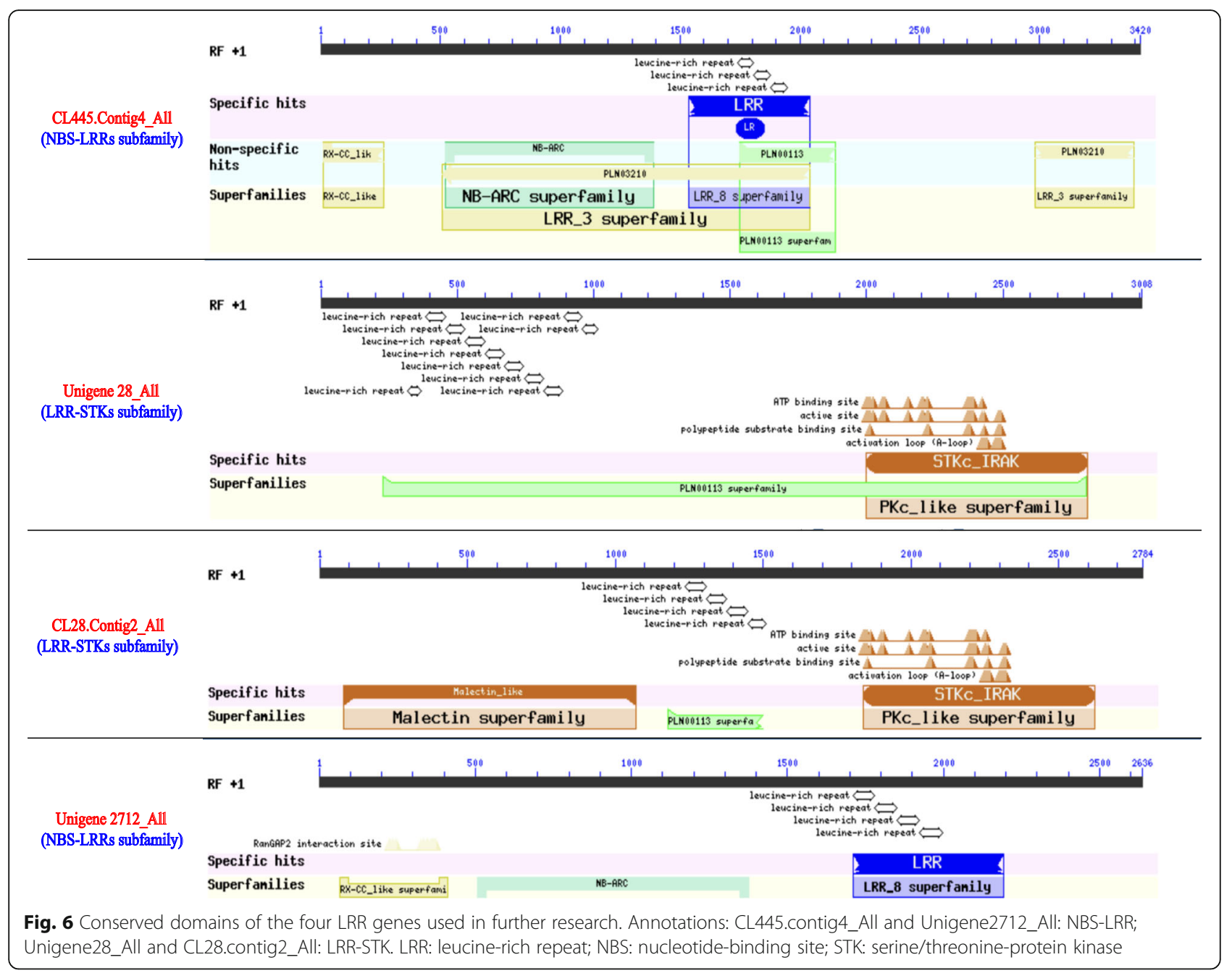

resistance to pathogens $[60,61]$. Research has shown that SA accumulation was increased in infected leaves and uninfected leaves that mediated systemic acquired resistance (SAR) [62]. SA accumulation can parallel the increase in pathogenesis-related protein gene expression [61]. For example, BAK1 overexpression caused SA accumulation and deregulation of cell death control genes [63].

JAs, including jasmonic acid and the MeJA derivatives, have proved effective at improving plant stress tolerance [64]. It was reported that JAs are key signaling compounds in hypersensitive response cell death and accumulate in response to bacterial flg22 in the grape Vitis rupestris [65].

Similar to SA and JAs, ABA (a weak acid that contains 15 carbon atoms) is an important stress hormone and is involved in physiological processes [66]. ABA participates in embryogenesis, seed germination, floral transition, and so on $[67,68]$.
However, plant growth, development, and stress responses need multiple phytohormones involved in complex signaling network crosstalk [69]. In this study, pitaya stems were treated with SA, MeJA, and ABA. Although all four genes responded to the three hormones, CL28.Contig2_All expression reached a peak at $48 \mathrm{~h}$ after SA treatment and Unigene28_All was clearly regulated by ABA at $2 \mathrm{~h}$. The results of hormones treatments showed that the expression of LRR family genes was regulated by plant hormones. The LRR family resistant genes and hormones related genes probably form a cross network to regulated plant disease together. Taking all the expression results together, we selected these two genes for further functional research. The subsequent analysis of conserved functional motifs in Unigene28_All and CL28.Contog2 All indicated that these two genes both have the typical LxxLxLxxNxL domain and the STRVGTIGYMAPE or SSVAxGTL/VGYLDPE conserved sites of STKc-IRAK (x denotes any aa) (Fig. 11). 


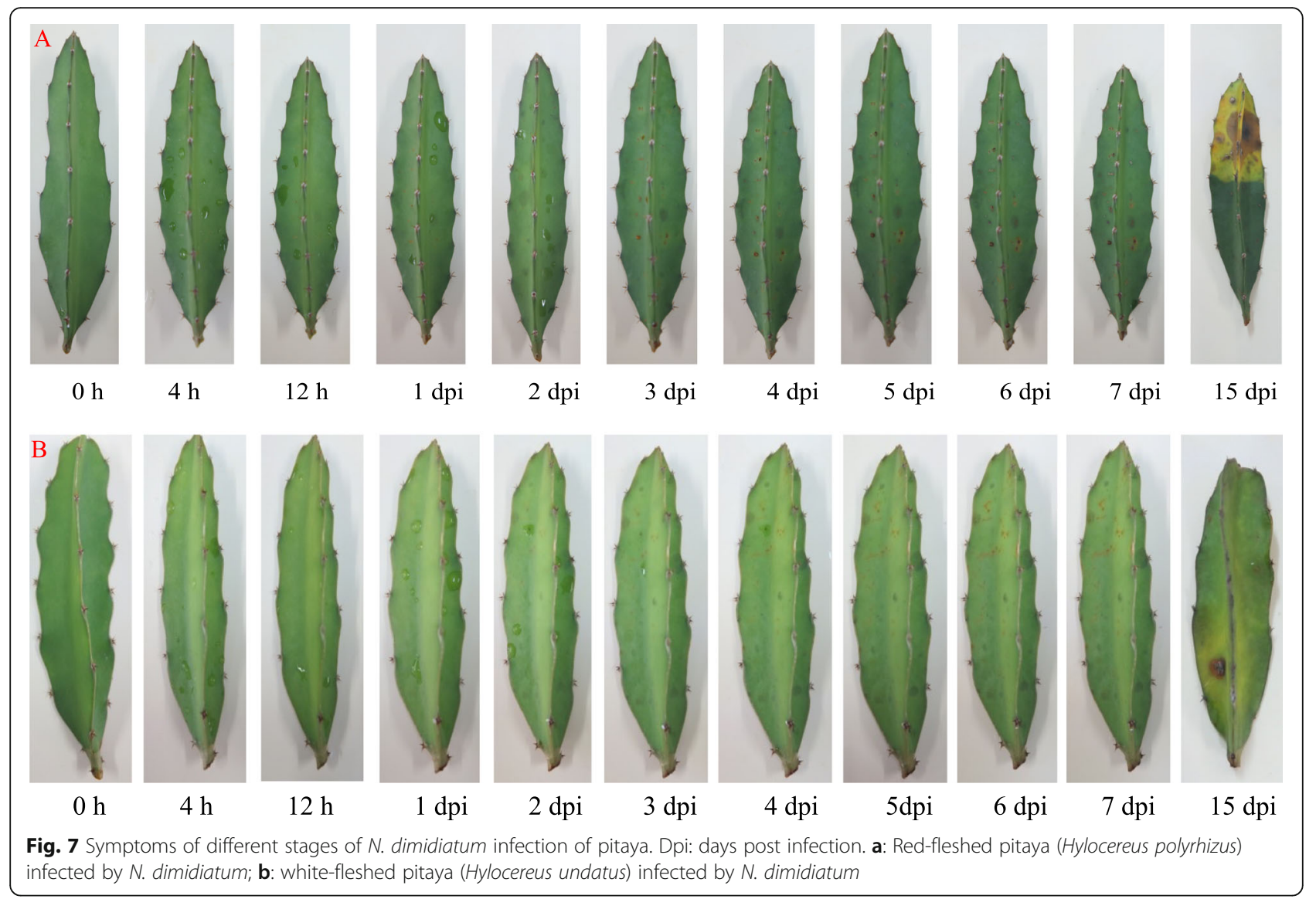

These domains or conserved sites may play important roles in protein function and signaling. However, in some cases, an LRR protein requires another helper or partner protein for functionality, so a complete functional study (including screening for interacting proteins) regarding Unigene28_All and CL28.Contog2_All is being carried out and the results will be published in the future.

Canker disease caused by $N$. dimidiatum is one of the most serious diseases of pitaya in the main growing regions [44]. The $N$. dimidiatum is a fungal which has a strong specificity infection of its host pitaya. Currently, because there is a very hard technological bottleneck in genetic transformation system of pitaya, it is very difficult to investigate the gene function in pitaya by transgenic methods just like in model plants. The fungal also can't infect the model plants like Arabidopsis thaliana, tobacco and tomato by our exploratory testing. So, it is a very hard work to identify the genes function with really deep experiments. At present, there are no reports on transcriptomics, molecular characterization, and expression analyses of LRR genes in pitaya induced by $N$. dimidiatum infection. From the expression profiles, we can identify the $H p L R R$ genes in response to pitaya canker disease preliminary. These results provide important research information for a relatively poor studied pathosystem at this moment, provide a framework for further functional investigation of the pitaya LRR subfamilies, and contribute to a better understanding of the complexity of LRR genes in higher plants.

\section{Conclusions}

Canker disease caused by the fungus $N$. dimidiatum is one of the most important diseases because of its rapid spread and strong specificity infection in its host pitaya. The occurrence of canker disease not only poses a significant threat in yields but also affects the quality of pitaya. Currently, it is an important job to excavate the resistant genes and explain the functions of them. This study mainly analyzed the $H p L R R$ family resistant genes structures by bioinformatics analysis and expression profiles in different pitaya tissues, different infected stages of red-fleshed pitaya (Hylocereus polyrhizus) and white-fleshed pitaya (Hylocereus undatus), and plant hormone treatments of $H p L R R$ family resistant genes. Results of this study provided a comprehensive overview of the $H p L R R$ family genes at transcriptional level in pitaya in 

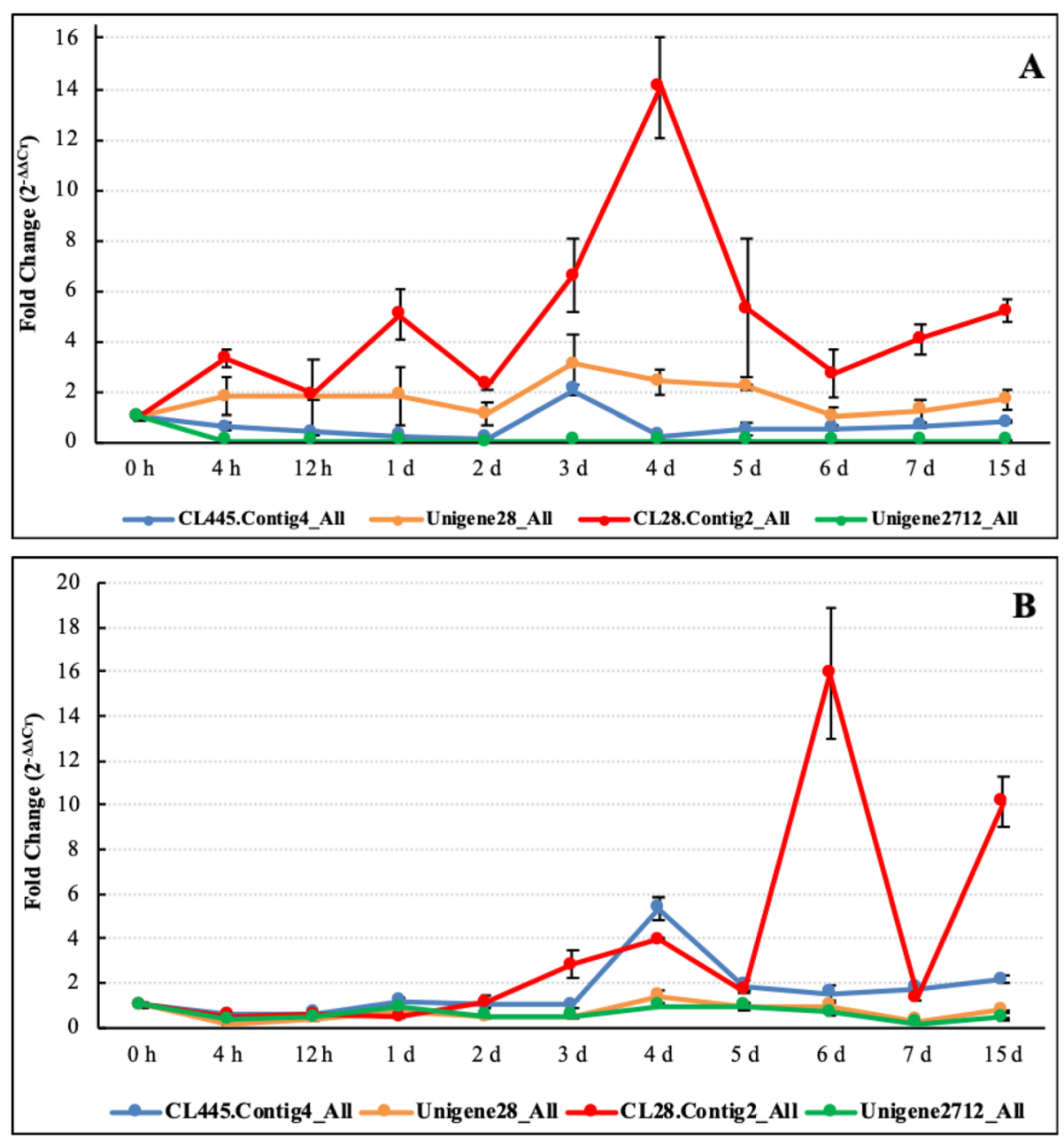

Fig. 8 Expression profiles of four HpLRR transcriptional genes at different stages and different pitaya species of $N$. dimidiatum infection. (a): HpLRR genesexpression profiles in red-fleshed pitaya (Hylocereus polyrhizus) infected by N. dimidiatum; (b): HpLRR genes expression profiles in whitefleshed pitaya(Hylocereus undatus) infected by $N$. dimidiatum. The $X$-axis represents different stages of $N$. dimidiatum infection and the $Y$-axis represents the foldchange $\left(2^{-\Delta \Delta C T}\right)$ according to qRT-PCR. Annotations: CL445.Contig4_All and Unigene2712_All: NBS-LRR; Unigene28_All and CL28.Contig2_All: LRRSTK. LRR: leucine-rich repeat; NBS: nucleotide-binding site; STK: serine/threonine-protein kinase

response to $N$. dimidiatum infection and provided a basis for further in-depth functional studies.

\section{Methods}

\section{Plant materials and growth conditions}

The normal (N1, N2, and N3) and diseased (D1, D2, and D3) tissues of pitaya (Hylocereus polyrhizus) stems used for RNA-seq were collected from Ledong County, Hainan Province, China that had been grown for about 5 years under natural conditions on a plantation. The normal $(\mathrm{N})$ group were healthy plants while the disease (D) group were diseased plants infected by $N$. dimidiatum fungal. The pitaya stems and fruits which infected by $N$. dimidiatum fungal appear small yellow spot initially, then spread quickly and almost all of the stems will go to rot finally. De novo transcriptome assembly and RNA-Seq were conducted using an Illumina HiSeq
System by Shenzhen BGI Tech Company (Shenzhen, China). Principal component analysis (PCA) and correlations analysis were used to evaluate the repeatability of samples. After analysis, the N1 and D2 samples were abandoned due to poor repeatability. Therefore, the bioinformatics analysis and follow-up experiments were carried out based on four samples (N2, N3, D1, and D3). The accession number of RNA-sequencing data and profiles was GSE119976 in Gene Expression Omnibus database of NCBI. In addition, the pitaya stem tissues for $N$. dimidiatum fungal infection, hormone treatment and tissues specific expression were collected in a plantation of Hainan University not same with those used for RNA-seq. Five pitaya plants were used in each condition and displayed similar symptoms. All five pitaya plants were merged together to use for qRT-PCR experiments. 


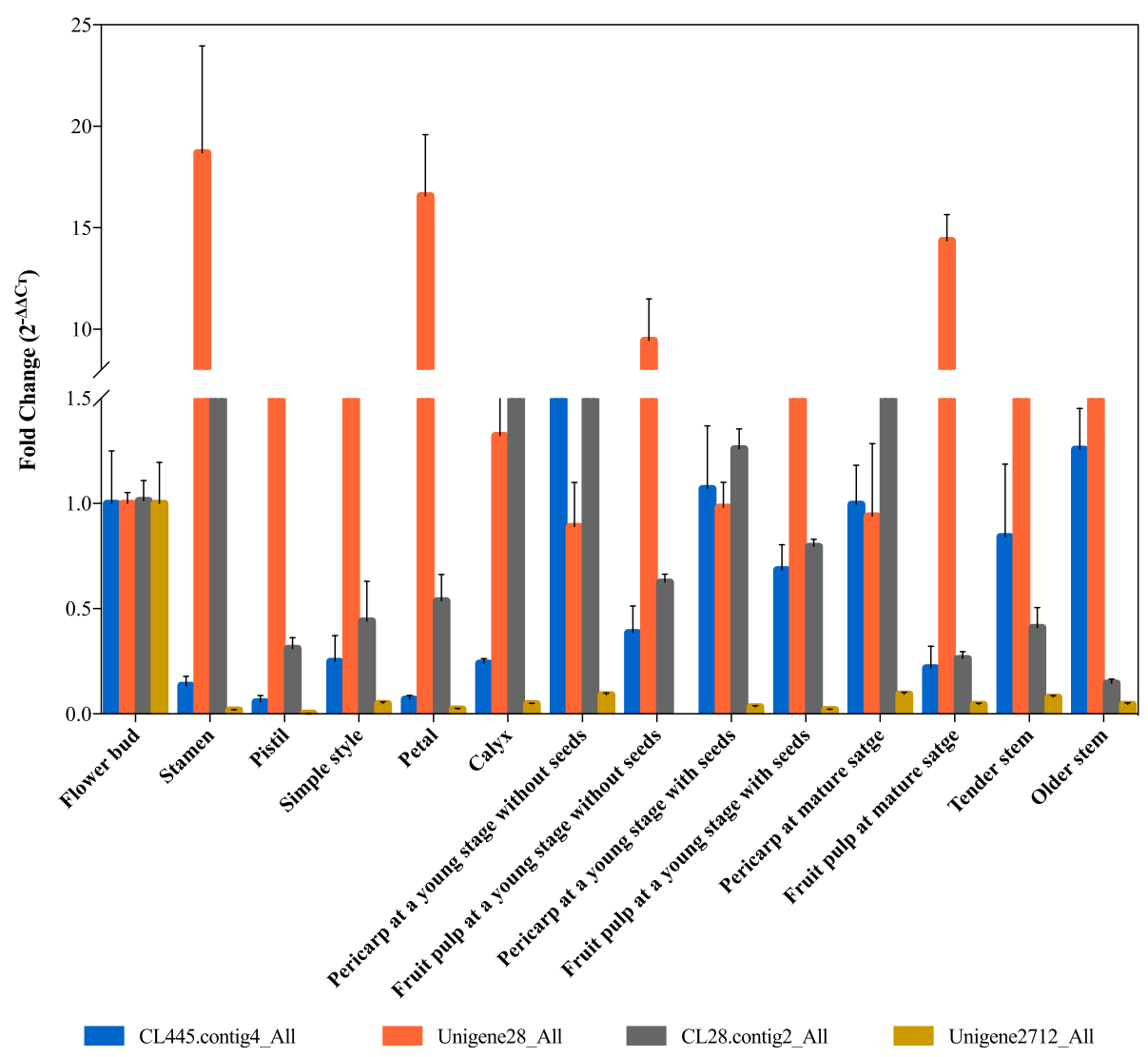

Fig. 9 Expression profiles of four HpLRR genes in different pitaya tissues. The $X$-axis represents different pitaya tissues and the $Y$-axis represents the fold change $\left(2^{-\triangle \Delta C T}\right)$ according to qRT-PCR. Annotations: CL445.contig4_All and Unigene2712_All: NBS-LRR; Unigene28_All and CL28.contig2_All: LRR-STK. LRR: leucine-rich repeat; NBS: nucleotide-binding site; STK: serine/threonine-protein kinase

Sequences assembled and RNA-Seq bioinformatics analysis Clean data was used for bioinformatics analysis which obtained by filtering from sequenced raw data [45]. Trinity paired-end assembly method was used to identify pitaya $H p L R R$ family genes [70]. TIGR Gene Indices clustering tools was used to obtain unigenes by clustering the assembled clean reads and eliminating redundancy [71]. These unigenes were blasted to public proteins databases to annotate and analyze. Which including. The Expectation Maximization (RSEM) software (Version: V1.2.12; Parameter: default; http://deweylab.biostat.wisc.edu/ RSEM) was used to obtain expression level expressed in FPKM. The calculation method of unigenes' FPKM was described as Li B [72]. The up- or down-regulated unigenes with an adjusted $P$-value $\leq 0.05$ and fold change $|(\log 2 \mathrm{FC}) \geq 1|$ were defined as differentially expressed genes (DEGs) using NOIseq and PossionDis methods.

\section{Identification and phylogenetic analysis of pitaya $H p L R R$ transcriptional genes}

The assembled unigenes were blasted to $\mathrm{nr}, \mathrm{GO}$, KEGG, COG, InterPro and SwissProt databases and the unigenes annotated to LRR family genes were selected for the next analysis. The expression levels of all $H p$ LRR genes were analyzed by heatmap analysis using the free online data analysis platform OmicShare tools (http://www.omicshare.com/tools/, Figs. 1 and 2). Thereafter, all the sequences of 233 LRR genes with coding sequences (CDSs) were used to construct a phylogenetic tree based on 1000 bootstrap replicates and the neighbor-joining (NJ) method using MEGA 6.0 software [46].

\section{Gene structure and phylogenetic analysis of $33 \mathrm{HpLRR}$ transcriptional genes with CDSs $>1.0 \mathrm{~kb}$}

There were 33 HpLRR transcriptional genes with CDSs $>1.0 \mathrm{~kb}$. Gene structure analysis of these genes was performed using the free online platform Gene Structure Display Server (GSDS v2.0, http://gsds.cbi. pku.edu.cn) by uploading the genes' CDSs and assembly sequences. Conserved motifs analysis was carried on MEME program based on the amino acid sequences of the CDS. The phylogenetic analysis was carried out based on 1000 bootstrap replicates and 

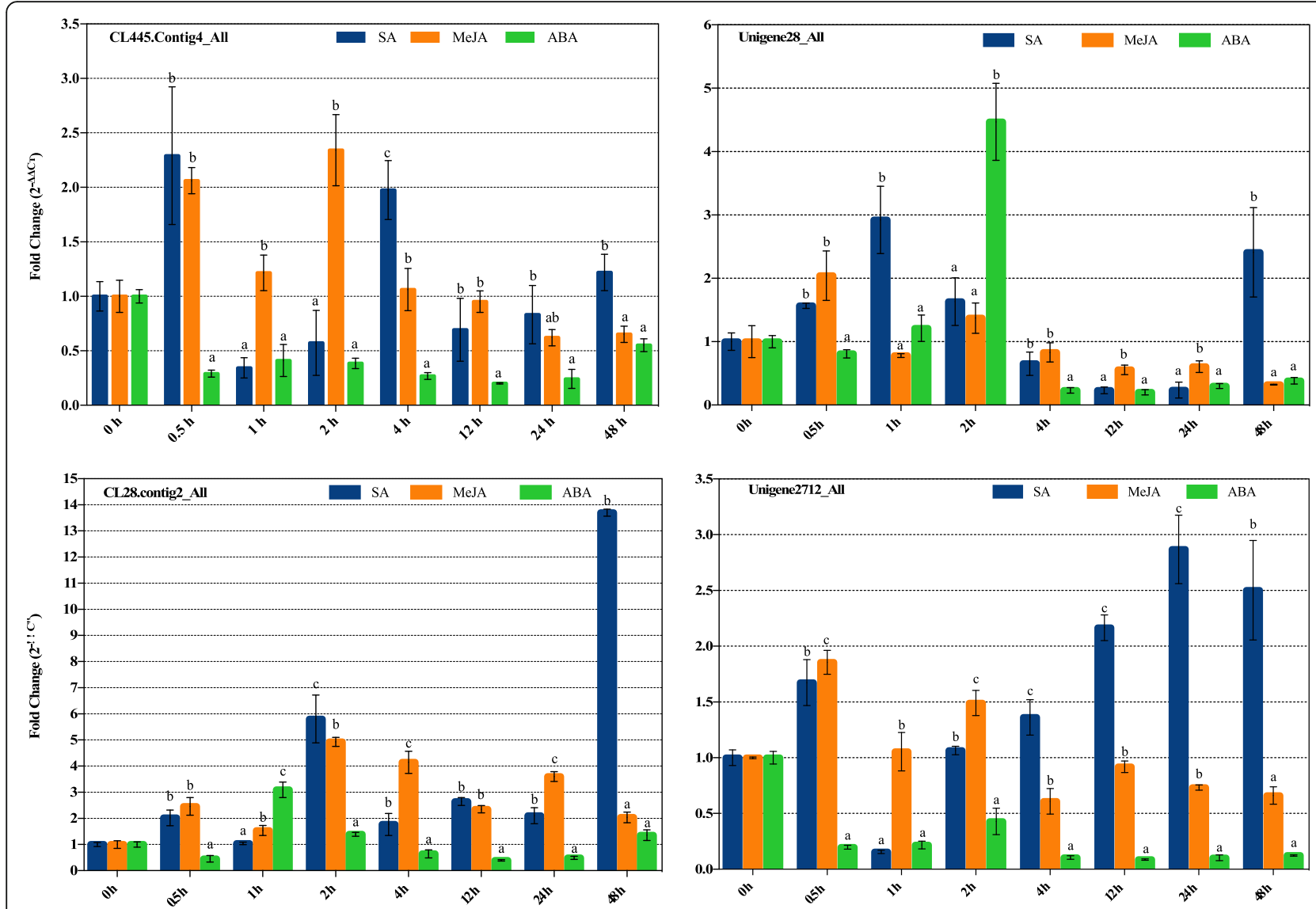

Fig. 10 Expression profiles of four HpLRR genes in response to SA, MeJA and ABA treatment. Annotations: CL445.contig4_All and Unigene2712_All: NBS-LRR; Unigene28_All and CL28.contig2_All: LRR-STK. LRR: leucine-rich repeat; NBS: nucleotide-binding site; STK: serine/ threonine-protein kinase. Different letters $(a, b, c)$ indicate significant difference of the expression of the target gene based on three technological replications $[P<0.05$, Single factor ANOVA test ( $F$ test), $n=3]$

the neighbor-joining (NJ) method using MEGA 6.0 software [46].

\section{Verification of 12 significantly up-regulated differentially expressed LRR genes (DEGs) by quantitative reverse transcription (qRT)-PCR}

In order to verify the reliability and consistency of the up or down regulated genes, all of the 12 differentially expressed LRR genes based on a PossionDis analysis were selected to perform qRT-PCR assay. The total RNA extraction of N2, N3, D1 and D3 pitaya samples were used improved cetyltrimethylammonium bromide (CTAB) method [73]. First strand cDNA synthesis of RNA was conducted according to manufacturer's protocols (No.6210A, Takara, Japan) after treated with DNase I (Thermo Fisher Scientific, USA). Then, the N2 and N3 cDNA template were balanced mixed together to obtain total N sample, same with D1 and D3 to obtain total D sample. qRT-PCR assay was performed using a $20-\mu \mathrm{L}$ reaction system according to the manufacturer's protocols of ChamQ $^{\text {m }}$ Universal SYBR $^{\circ}$ qPCR Master Mix kit (Q711-02/03, Vazyme Biotech Co., Ltd., Beijing, China). Twelve significantly up-regulated $H p$ LRR family genes were selected for qRT-PCR assays using a 7500 Applied Biosystems qRT-PCR System (Life Tech, 81 Wyman Street, Waltham, MA, 02454, USA). It was proved that the pitaya ubiquitin gene $(U B Q)$ was an great internal reference for data normalization [74]. The $12 \mathrm{HpLRR}$ family genes and $U B Q$ primers were designed using Primer Premier 6 software (www.premierbiosoft.com). The qRT-PCR conditions were performed as previous study [45]. The Delta-Delta cycle threshold $\left(2^{-\Delta \Delta C T}\right)$ method was used to output data analysis based on three technical replicates.

\section{Expression profiles of four HpLRR transcriptional genes under different stages of $\boldsymbol{N}$. dimidiatum infection in different pitaya species}

Among the total set of LRR genes, four $H p L R R$ transcriptional genes (CL445.Contig4_All, Unigene 28_All, 


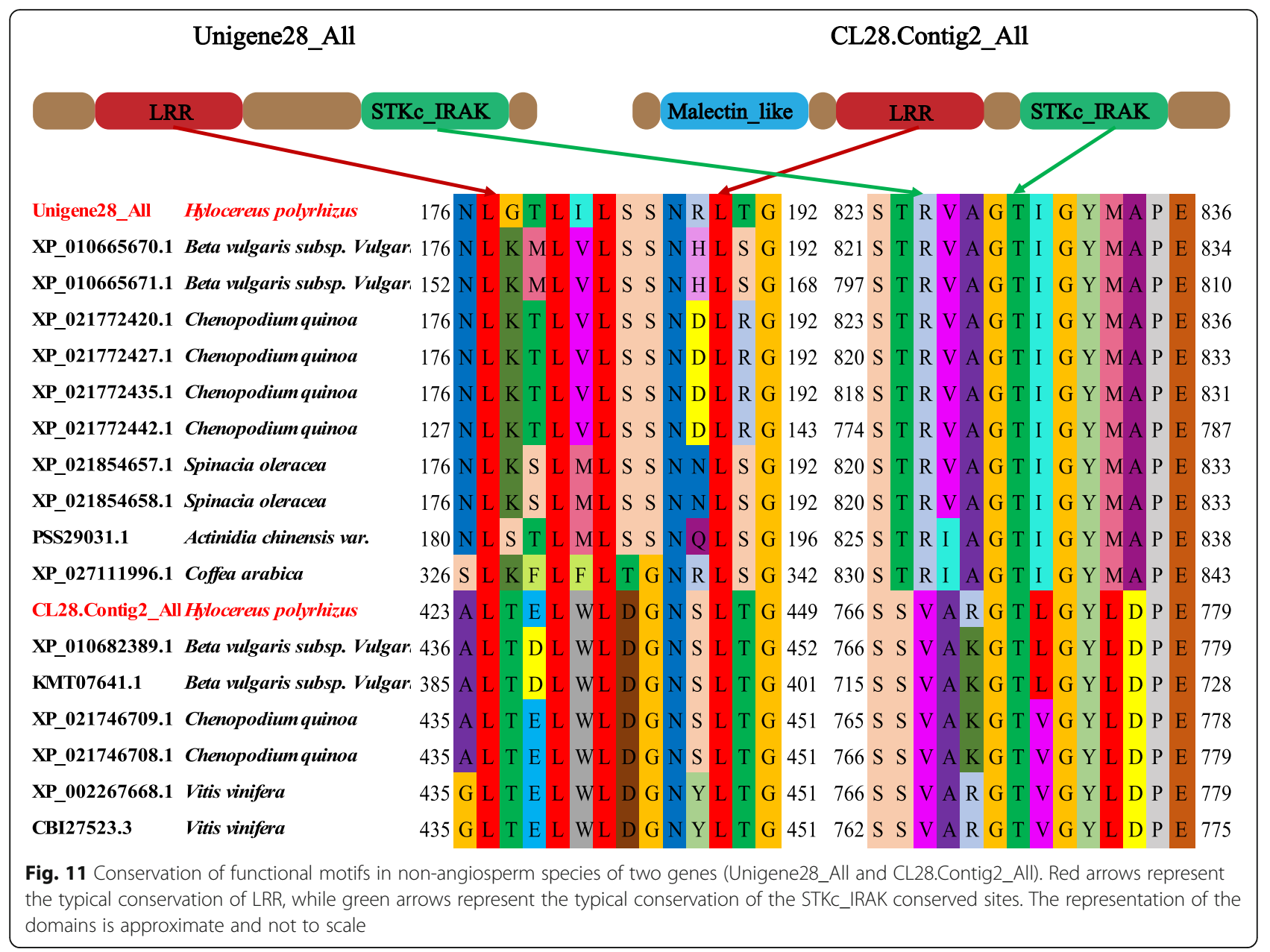

CL28.Contig2_All, and Unigene 2712_All) were selected for further expression research as they had the four longest CDSs. Red-fleshed pitaya (Hylocereus polyrhizus) "Jinduyihao" and white-fleshed pitaya (Hylocereus undatus) "Vietnam Bairou" tender stems were used for $N$. dimidiatum infection. The 5 healthy individual pitaya plants were used as control which collected from a plantation in Hainan University. Another 5 healthy individual pitaya plants were used for infected. The infected experiments were performed as following: $N$. dimidiatum hypha were collected after growing them in potato dextrose agar medium for 7-10 days. Before inoculation, the stems were sterilized twice using $75 \%$ ethyl alcohol. The hyphae were diluted, with 20-40 conidia in suspension, using 400X microscope magnification (Olympus Corporation, Tokyo, Japan). Inoculation was conducted by spraying the conidial suspension onto the pitaya stems. After spraying, stems were wrapped in absorbent wool with distilled water to retain moisture. The plants were then placed in an illuminated incubator at $28^{\circ} \mathrm{C}$ under a $16 / 8 \mathrm{~h}$ light/dark cycle for 15 days. The pitaya stem tissues were collected for total RNA extraction after $0 \mathrm{~h}, 4 \mathrm{~h}, 12 \mathrm{~h}, 1$ day, 2 days, 3 days, 4 days, 5 days, 6 days, 7 days, and 15 days of $N$. dimidiatum infection. The total RNA was reversed transcribed into cDNA and the cDNA was used for qRT-PCR (as in the previous steps). The qRT-PCR primers for the four LRR genes were designed using Primer Premier 6.0 software (www.premierbiosoft.com). The primer details for the four genes are presented in Supplenmentary Information 1, 2, 3, 4.

\section{Expression profiles of the four HpLRR genes in different tissues of pitaya}

The following 14 healthy pitaya tissues were studied: (1) flower bud, (2) stamen, (3) pistil, (4) simple style, (5) petal, (6) calyx, (7-12) pericarp and fruit pulp of a young green, mature green, and red fruit, (13) tender stems, and (14) older stems were collected in pitaya plantation, Hainan province. The total RNA of the 14 tissues was extracted, reversed transcribed into cDNA, and used for 
qRT-PCR to obtain the expression profiles of the four selected genes.

\section{Response of the four HpLRR genes to salicylic acid (SA), methyl jasmonate (MeJA), and abscisic acid (ABA) treatments}

Healthy pitaya stems were collected from the plantation and sterilized twice using 75\% ethyl alcohol before being treated with $5 \mathrm{mM}$ SA solution (pH 5.7), $1 \mathrm{mM} \mathrm{MeJA} \mathrm{so-}$ lution, and $100 \mathrm{uM} \mathrm{ABA}$ solution. The three solutions were all added with $0.01 \%$ Silwet-77 (Code: DE0025, Beijing BioDee Biotechnology Co. Ltd., China) to reduce the water's surface tension. The stem tissues were collected after $0 \mathrm{~h}, 0.5 \mathrm{~h}, 1 \mathrm{~h}, 2 \mathrm{~h}, 4 \mathrm{~h}, 12 \mathrm{~h}, 24 \mathrm{~h}$, and $48 \mathrm{~h}$ of hormone treatment. RNA extraction and qRT-PCR assays were performed as in the previous steps.

\section{Supplementary information}

Supplementary information accompanies this paper at https://doi.org/10. 1186/s12870-020-02368-6.

Additional file 1 Supplenmentary Information 1: Information of all of the 272 genes.

Additional file $\mathbf{2}$ Supplenmentary Information 2: Details information about the sequence characteristics of 233 LRR related-genes with the longest CDS.

Additional file 3 Supplenmentary Information 3: Primers used for the genes in the manuscript.

Additional file 4 Supplenmentary Information 4: Sequences were identified as LRR family genes on each database.

\section{Abbreviations}

LRRs: Leucine-rich repeat genes; LRR-RLKs: Leucine-rich repeats receptor-like kinases; NBS-LRRs: Nucleotide binding site leucine-rich repeats; LRRSTKs: Leucine-rich repeats receptor-like serine/threonine-protein kinases: BRI1: Brassinosteroid-insensitive 1; BAK1: BRI1-associated receptor kinase 1; NB-ARC: Nucleotide binding adaptor shared by Apaf-1 resistance proteins, CED-4 domain; CC-NBS-LRR: Coiled-coil motif nucleotide-binding site leucine-rich repeat; SA: Salicylic acid; JA: Jasmonates; MeJA: Methyl jasmonate; ABA: Abscisic acid; SAR: Systemic acquired resistance; PR: Pathogenesis related protein

\section{Acknowledgements}

We thank professor Yongchuan Deng in Hainan University for providing experimental help for us.

\section{Authors' contributions}

$\mathrm{TH}^{*}$ conceived the study and participated in the design of all experiments. $\mathrm{HJQ}^{*}$ took part in the experiments design and provided good idea during the research process, including the manuscript writing. XM performed the qRT-PCR analysis, statistical analysis and wrote the manuscript. LCL carried out RNA extraction, GRT-PCR validation and statistical analysis. FY, LZW and GPY conducted the bioinformatics studies. XR, CY and WSS participated in data analysis and discussion about the experiments. All authors read and approved the final manuscript.

\section{Funding}

This work was supported by the Construction of World First Class Discipline of Hainan University (RZZX201904), Species and Varieties Resources Protection Project of Ministry of Agriculture and Rural Affairs of China (18190033-1. Germplasm Protection of Pitaya), the Crop Science Postgraduate Innovation Project of Hainan University Tropical Agriculture and Forestry College (ZWCX2018007). The funders had no role in the design of the study, the collection, analysis, and interpretation of data and in writing the manuscript.

\section{Availability of data and materials}

The accession number of RNA-sequencing data and profiles was GSE119976 in Gene Expression Omnibus (GEO) database via NCBI website (https://www. ncbi.nlm.nih.gov/geo/query/acc.cgi?acc=GSE119976). The datasets generated and analyzed during the current study are available from the GEO website or corresponding author on reasonable request.

\section{Ethics approval and consent to participate}

Permissions were obtained from the pitaya orchard of Ledong Country, Hainan Province for the sampling and experiments used in this study.

\section{Consent for publication}

Not applicable.

\section{Competing interests}

The authors declare that they have no competing interests.

Received: 11 September 2019 Accepted: 27 March 2020

Published online: 15 April 2020

\section{References}

1. Thomma BP, Nurnberger $T$, Joosten MH. Of PAMPs and effectors: the blurred PTI-ETI dichotomy. Plant Cell. 2011;23(1):4-15.

2. Newman MA, Sundelin T, Nielsen JT, Erbs G. MAMP (microbe-associated molecular pattern) triggered immunity in plants. Front Plant Sci. 2013;4:139.

3. Zipfel C. Plant pattern-recognition receptors. Trends Immunol. 2014;35(7): $345-51$

4. Jurca ME, Bottka S, Feher A. Characterization of a family of Arabidopsis receptor-like cytoplasmic kinases (RLCK class VI). Plant Cell Rep. 2008;27(4): 739-48.

5. Greeff C, Roux M, Mundy J, Petersen M. Receptor-like kinase complexes in plant innate immunity. Front Plant Sci. 2012;3:209.

6. Liu T, Liu Z, Song C, Hu Y, Han Z, She J, Fan F, Wang J, Jin C, Chang J, et al. Chitin-induced dimerization activates a plant immune receptor. Science. 2012;336(6085):1160-4

7. Cao Y, Liang Y, Tanaka K, Nguyen CT, Jedrzejczak RP, Joachimiak A, Stacey G. The kinase LYK5 is a major chitin receptor in Arabidopsis and forms a chitin-induced complex with related kinase CERK1. eLife. 2014;3.

8. Lin W, Li B, Lu D, Chen S, Zhu N, He P, Shan L. Tyrosine phosphorylation of protein kinase complex BAK1/BIK1 mediates Arabidopsis innate immunity. Proc Natl Acad Sci U S A. 2014;111(9):3632-7.

9. Shi $H$, Shen Q, Qi Y, Yan $H$, Nie H, Chen Y, Zhao T, Katagiri F, Tang D. BR-SIGNALING KINASE1 physically associates with FLAGELLIN SENSING2 and regulates plant innate immunity in Arabidopsis. Plant Cell. 2013, 25(3):1143-57.

10. Kobe B, Deisenhofer J. Proteins with leucine-rich repeats. Curr Opin Struct Biol. 1995;5(3):409-16

11. Kim SH, Kwon SI, Saha D, Anyanwu NC, Gassmann W. Resistance to the Pseudomonas syringae effector HopA1 is governed by the TIR-NBS-LRR protein RPS6 and is enhanced by mutations in SRFR1. Plant Physiol. 2009; 150(4):1723-32.

12. Van der Biezen EA, Jones JD. Plant disease-resistance proteins and the gene-for-gene concept. Trends Biochem Sci. 1998;23(12):454-6.

13. Sekhwal MK, Li P, Lam I, Wang X, Cloutier S, You FM. Disease resistance gene analogs (RGAs) in plants. Int J Mol Sci. 2015;16(8):19248-90.

14. Song D, Li G, Song F, Zheng Z. Molecular characterization and expression analysis of OSBISERK1, a gene encoding a leucine-rich repeat receptor-like kinase, during disease resistance responses in rice. Mol Biol Rep. 2008;35(2): 275-83.

15. Bella J, Hindle KL, McEwan PA, Lovell SC. The leucine-rich repeat structure Cell Mol Life Sci. 2008;65(15):2307-33

16. Williams SJ, Yin L, Foley G, Casey LW, Outram MA, Ericsson DJ, Lu J, Boden M, Dry IB, Kobe B. Structure and function of the TIR domain from the grape NLR protein RPV1. Front Plant Sci. 2016;7:1850

17. Gou X, He K, Yang H, Yuan T, Lin H, Clouse SD, Li J. Genome-wide cloning and sequence analysis of leucine-rich repeat receptor-like protein kinase genes in Arabidopsis thaliana. BMC Genomics. 2010;11:19. 
18. Chinchilla D, Bauer Z, Regenass M, Boller T, Felix G. The Arabidopsis receptor kinase FLS2 binds flg22 and determines the specificity of flagellin perception. Plant Cell. 2006;18(2):465-76.

19. Hong JK, Hwang IS, Hwang BK. Functional roles of the pepper leucine-rich repeat protein and its interactions with pathogenesis-related and hypersensitive-induced proteins in plant cell death and immunity. Planta. 2017;246(3):351-64.

20. Cheng W, Xiao Z, Cai H, Wang C, Hu Y, Xiao Y, Zheng Y, Shen L, Yang S, Liu $Z$, et al. A novel leucine-rich repeat protein, CaLRR51, acts as a positive regulator in the response of pepper to Ralstonia solanacearum infection. Mol Plant Pathol. 2017;18(8):1089-100.

21. Afzal AJ, Wood AJ, Lightfoot DA. Plant receptor-like serine threonine kinases: roles in signaling and plant defense. Mol Plant Microbe Interact. 2008;21(5): 507-17.

22. Li J, Wen J, Lease KA, Doke JT, Tax FE, Walker JC. BAK1, an Arabidopsis LRR receptor-like protein kinase, interacts with $\mathrm{BRI1}$ and modulates brassinosteroid signaling. Cell. 2002;110(2):213-22.

23. Nam KH, Li J. BRI1/BAK1, a receptor kinase pair mediating brassinosteroid signaling. Cell. 2002;110(2):203-12.

24. Albert I, Zhang L, Bemm H, Nurnberger T. Structure-function analysis of immune receptor AtRLP23 with its ligand nlp20 and Coreceptors AtSOBIR and AtBAK1. Mol Plant Microbe Interac. 2019;32(8):1038-46.

25. Cui HR, Zhang ZR, Lv W, Xu JN, Wang XY. Genome-wide characterization and analysis of F-box protein-encoding genes in the Malus domestica genome. Mol Genet Genomics. 2015;290(4):1435-46.

26. Kuchay S, Duan SS, Schenkein E, Peschiaroli A, Saraf A, Florens L, Washburn MP, Pagano M. FBXL2-and PTPL1-mediated degradation of p110-free p85 beta regulatory subunit controls the PI (3) K signalling cascade. Nat Cell Biol. 2013;15(5):472

27. Jia Q, Xiao ZX, Wong FL, Sun S, Liang KJ, Lam HM: Genome-Wide Analyses of the Soybean F-Box Gene Family in Response to Salt Stress. Int J Mol Sci. 2017;18(4):818-34

28. Song JB, Wang YX, Li HB, Li BW, Zhou ZS, Gao S, Yang ZM. The F-box family genes as key elements in response to salt, heavy mental, and drought stresses in Medicago truncatula. Funct Integr Genomics. 2015; 15(4):495-507.

29. Hu Z, Keceli MA, Piisila M, Li J, Survila M, Heino P, Brader G, Palva ET, Li J. Fbox protein AFB4 plays a crucial role in plant growth, development and innate immunity. Cell Res. 2012;22(4):777-81.

30. Angot A, Peeters N, Lechner E, Vailleau F, Baud C, Gentzbittel L, Sartorel E, Genschik P, Boucher C, Genin S. Ralstonia solanacearum requires F-box-like domain-containing type III effectors to promote disease on several host plants. Proc Natl Acad Sci U S A. 2006;103(39):14620-5.

31. Li LQ, Pan D, Chen H, Zhang L, Xie WJ. F-box protein FBXL2 inhibits gastric cancer proliferation by ubiquitin-mediated degradation of forkhead box M1. FEBS Lett. 2016;590(4):445-52.

32. Chen BB, Glasser JR, Coon TA, Mallampalli RK. Skp-cullin-F box E3 ligase component FBXL2 ubiquitinates Aurora B to inhibit tumorigenesis. Cell Death Dis. 2013:4:e759.

33. Tosto G, Fu H, Vardarajan BN, Lee JH, Cheng R, Reyes-Dumeyer D, Lantigua R, Medrano M, Jimenez-Velazquez IZ, Elkind MS, et al. F-box/LRR-repeat protein 7 is genetically associated with Alzheimer's disease. Ann Clin Transl Neurol. 2015;2(8):810-20.

34. Tran DTN, Chung EH, Habring-Muller A, Demar M, Schwab R, Dangl JL, Weigel D, Chae E. Activation of a plant NLR complex through Heteromeric association with an autoimmune risk variant of another NLR. Curr Biol. 2017; 27(8):1148-60

35. Takken FL, Albrecht M, Tameling WI. Resistance proteins: molecular switches of plant defence. Curr Opin Plant Biol. 2006;9(4):383-90.

36. Bentham A, Burdett $H$, Anderson PA, Williams SJ, Kobe B. Animal NLRs provide structural insights into plant NLR function. Ann Bot. 2017;119(5): 827-702.

37. Chakraborty J, Jain A, Mukherjee D, Ghosh S, Das S. Functional diversification of structurally alike NLR proteins in plants. Plant Sci. 2018;269: 85-93.

38. Zhao Y, Weng Q, Song J, Ma H, Yuan J, Dong Z, Liu Y. Bioinformatics analysis of NBS-LRR encoding resistance genes in Setaria italica. Biochem Genet. 2016:54(3):232-48

39. Zhu X, Lu C, Du L, Ye X, Liu X, Coules A, Zhang Z. The wheat NB-LRR gene TaRCR1 is required for host defence response to the necrotrophic fungal pathogen Rhizoctonia cerealis. Plant Biotechnol J. 2017;15(6):674-87.
40. Xing L, Hu P, Liu J, Witek K, Zhou S, Xu J, Zhou W, Gao L, Huang Z, Zhang R, et al. Pm21 from Haynaldia villosa encodes a CC-NBS-LRR protein conferring powdery mildew resistance in wheat. Mol Plant. 2018;11(6):874-8.

41. Li X, Zhang Y, Yin L, Lu J. Overexpression of pathogen-induced grapevine TIR-NB-LRR gene VaRGA1 enhances disease resistance and drought and salt tolerance in Nicotiana benthamiana. Protoplasma. 2017;254(2):957-69.

42. Xun $H$, Yang $X$, He H, Wang M, Guo P, Wang Y, Pang J, Dong Y, Feng $X$, Wang $S$, et al. Over-expression of GmKR3, a TIR-NBS-LRR type R gene, confers resistance to multiple viruses in soybean. Plant Mol Biol. 2019;99(12):95-111.

43. Chuang MF, Ni HF, Yang HR, Shu SL, Lai SY, Jiang YL. First report of stem canker disease of pitaya (Hylocereus undatus and $\mathrm{H}$. polyrhizus) caused by Neoscytalidium dimidiatum in Taiwan. Plant Dis. 2012;96(6):906

44. Xu M, Peng Y, Qi Z, Yan Z, Yang L, He M-D, Li Q-X, Liu C-L, Ruan Y-Z, Wei S$S$, et al. Identification of Neoscytalidium dimidiatum causing canker disease of pitaya in Hainan, China. Australas Plant Pathol. 2018;47(5):547-53.

45. Xu M, Liu CL, Luo J, Qi Z, Yan Z, Fu Y, Wei SS, Tang H. Transcriptomic de novo analysis of pitaya (Hylocereus polyrhizus) canker disease caused by Neoscytalidium dimidiatum. BMC Genomics. 2019;20(1):10.

46. Tamura K, Stecher G, Peterson D, Filipski A, Kumar S. MEGA6: molecular evolutionary genetics analysis version 6.0. Mol Biol Evol. 2013;30(12):2725-9.

47. Schallus T, Jaeckh C, Feher K, Palma AS, Liu Y, Simpson JC, Mackeen M, Stier G, Gibson TJ, Feizi T, et al. Malectin: a novel carbohydrate-binding protein of the endoplasmic reticulum and a candidate player in the early steps of protein N-glycosylation. Mol Biol Cell. 2008;19(8):3404-14.

48. Robert-Seilaniantz A, Navarro L, Bari R, Jones JD. Pathological hormone imbalances. Curr Opin Plant Biol. 2007;10(4):372-9.

49. Padmanabhan M, Cournoyer P, Dinesh-Kumar SP. The leucine-rich repeat domain in plant innate immunity: a wealth of possibilities. Cell Microbiol. 2009:11(2):191-8.

50. Kang UB, Marto JA. Leucine-rich repeat kinase 2 and Parkinson's disease. Proteomics. 2017;17:1-2

51. Hugot JP, Chamaillard M, Zouali H, Lesage S, Cezard JP, Belaiche J, Almer S, Tysk C, O'Morain CA, Gassull M, et al. Association of NOD2 leucine-rich repeat variants with susceptibility to Crohn's disease. Nature. 2001;411(6837):599-603.

52. Dangl JL, Jones JD. Plant pathogens and integrated defence responses to infection. Nature. 2001;411(6839):826-33.

53. Forsthoefel NR, Dao TP, Vernon DM. PIRL1 and PIRL9, encoding members of a novel plant-specific family of leucine-rich repeat proteins, are essential for differentiation of microspores into pollen. Planta. 2010;232(5):1101-14.

54. Torii KU. Leucine-rich repeat receptor kinases in plants: structure, function, and signal transduction pathways. Int Rev Cytol. 2004;234:1-46.

55. Chaparro-Garcia A, Wilkinson RC, Gimenez-lbanez S, Findlay K, Coffey MD, Zipfel C, Rathjen JP, Kamoun S, Schornack S. The receptor-like kinase SERK3/ BAK1 is required for basal resistance against the late blight pathogen phytophthora infestans in Nicotiana benthamiana. PLoS One. 2011;6(1):e16608.

56. Clark SE, Running MP, Meyerowitz EM. CLAVATA1, a regulator of meristem and flower development in Arabidopsis. Development. 1993;119(2):397-418.

57. Jeong S, Trotochaud AE, Clark SE. The Arabidopsis CLAVATA2 gene encodes a receptor-like protein required for the stability of the CLAVATA1 receptorlike kinase. Plant Cell. 1999;11(10):1925-34.

58. Rojo E, Sharma VK, Kovaleva V, Raikhel NV, Fletcher JC. CLV3 is localized to the extracellular space, where it activates the Arabidopsis CLAVATA stem cell signaling pathway. Plant Cell. 2002;14(5):969-77.

59. Liu W, Liu J, Triplett L, Leach JE, Wang GL. Novel insights into rice innate immunity against bacterial and fungal pathogens. Annu Rev Phytopathol. 2014:52:213-41.

60. Yi SY, Kwon SY. How does SA signaling link the Flg22 responses? Plant Signal Behav. 2014;9(11):e972806.

61. An C, Mou Z. Salicylic acid and its function in plant immunity. J Integr Plant Biol. 2011;53(6):412-28.

62. Aarts N, Metz M, Holub E, Staskawicz BJ, Daniels MJ, Parker JE. Different requirements for EDS1 and NDR1 by disease resistance genes define at least two R gene-mediated signaling pathways in Arabidopsis. Proc Natl Acad Sci U S A. 1998;95(17):10306-11.

63. Kim SY, Shang Y, Joo SH, Kim SK, Nam KH. Overexpression of BAK1 causes salicylic acid accumulation and deregulation of cell death control genes. Biochem Biophys Res Commun. 2017:484(4):781-6.

64. Ahmad P, Rasool S, Gul A, Sheikh SA, Akram NA, Ashraf M, Kazi AM, Gucel S. Jasmonates: multifunctional roles in stress tolerance. Front Plant Sci. 2016;7:813. 
65. Chang X, Seo M, Takebayashi Y, Kamiya Y, Riemann M, Nick P. Jasmonates are induced by the PAMP flg22 but not the cell death-inducing elicitor Harpin in Vitis rupestris. Protoplasma. 2017;254(1):271-83.

66. Dong T, Park Y, Hwang I. Abscisic acid: biosynthesis, inactivation, homoeostasis and signalling. Essays Biochem. 2015;58:29-48.

67. Li Y, Wang C, Liu X, Song J, Li H, Sui Z, Zhang M, Fang S, Chu J, Xin M, et al. Up-regulating the abscisic acid inactivation gene ZmABA80x1b contributes to seed germination heterosis by promoting cell expansion. J Exp Bot. 2016 67(9):2889-900.

68. Yang W, Zhang W, Wang X. Post-translational control of ABA signalling: the roles of protein phosphorylation and ubiquitination. Plant Biotechnol J. 2017;15(1):4-14.

69. Ning Y, Liu W, Wang GL. Balancing immunity and yield in crop plants. Trends Plant Sci. 2017;22(12):1069-79.

70. Grabherr MG, Haas BJ, Yassour M, Levin JZ, Thompson DA, Amit I, Adiconis X, Fan L, Raychowdhury R, Zeng Q, et al. Full-length transcriptome assembly from RNA-Seq data without a reference genome. Nat Biotechnol. 2011;29(7): 644-52.

71. Pertea G, Huang X, Liang F, Antonescu V, Sultana R, Karamycheva S, Lee $Y$, White J, Cheung F, Parvizi B, et al. TIGR Gene Indices clustering tools (TGICL): a software system for fast clustering of large EST datasets. Bioinformatics. 2003;19(5):651-2.

72. Li B, Dewey CN. RSEM: accurate transcript quantification from RNA-Seq data with or without a reference genome. BMC Bioinformatics. 2011;12:323.

73. White EJ, Venter M, Hiten NF, Burger JT. Modified Cetyltrimethylammonium bromide method improves robustness and versatility: the benchmark for plant RNA extraction. Biotechnol J. 2008;3(11):1424-8.

74. Fengxia YXW, Guoli G, Jin T. Cloning and sequence analysis of housekeeping genes actin and UBQ from pitaya. Guizhou Agric Sci. 2013; 41(9):4.

\section{Publisher's Note}

Springer Nature remains neutral with regard to jurisdictional claims in published maps and institutional affiliations.

Ready to submit your research? Choose BMC and benefit from:

- fast, convenient online submission

- thorough peer review by experienced researchers in your field

- rapid publication on acceptance

- support for research data, including large and complex data types

- gold Open Access which fosters wider collaboration and increased citations

- maximum visibility for your research: over $100 \mathrm{M}$ website views per year

At $\mathrm{BMC}$, research is always in progress.

Learn more biomedcentral.com/submissions 